\title{
Monodromy of inhomogeneous Picard-Fuchs equations
}

\author{
Robert A. Jefferson And Johannes Walcher
}

\begin{abstract}
We study low-degree curves on one-parameter Calabi-Yau hypersurfaces, and their contribution to the space-time superpotential in a superstring compactification with D-branes. We identify all lines that are invariant under at least one permutation of the homogeneous variables, and calculate the inhomogeneous PicardFuchs equation. The irrational large volume expansions satisfy the recently discovered algebraic integrality. The bulk of our work is a careful study of the topological integrality of monodromy under navigation around the complex structure moduli space. This is a powerful method to recover the single undetermined integration constant that is itself also of arithmetic significance. The examples feature a variety of residue fields, both abelian and non-abelian extensions of the rationals, thereby providing a glimpse of the arithmetic D-brane landscape.
\end{abstract}

1. Introduction and nature of results

2. From curves to residues

2.1. Quintic

2.2. Sextic

2.3. Octic

2.4. Dectic

3. Algebraic integrality

3.1. Octic

3.1.1 First component 
3.2. Dectic

3.3. Quintic

4. Integrality of monodromy

4.1. Octic

4.1.1 First component

4.1.2 Second component

4.3. Quintic

5. Discussion

Acknowledgments

Appendix. Some details of residue calculation

References

\section{Introduction and nature of results}

The reader of this note will appreciate that when it comes to calculations around moduli spaces parameterizing supersymmetric vacua of quantum field theories and string theory, explicit evaluations of global monodromy rank among both the most subtle and the hardest. This is so because except in the simplest situations (really, anything that cannot be reduced to the thrice punctured sphere, or hypergeometric functions), the required analytic continuations cannot be handled algebraically, and one has to resort to numerical methods. (This would be even more true for higher dimensional moduli spaces.) Moreover, the precise matching of the local data from one patch to the next is contingent on keeping track of the chosen continuation path, and the relative normalization.

These facts notwithstanding, monodromy calculations are often a worthwhile enterprise. A priori knowledge of (even part of) the monodromy constitutes valuable information to constrain the behavior around the singular points which are of more direct physical and mathematical interest. A posteriori, consistent monodromies serve as cross-check of local results, and are 
the final confirmation that all normalizations are correct. In some situations, such as the one studied in the present paper, monodromy considerations can be used to determine subtle local data whose perturbative calculation is either much harder or even unknown. This will be the main payoff of the present paper.

Much of the physics motivation for the calculations that we will present flows from the realization that in the context of Calabi-Yau compactifications of type II string theory, breaking supersymmetry from eight to four supercharges by wrapping D-branes comes, at the level of solving the Fflatness equations on the worldvolume, with an extension of moduli spaces, schematically ${ }^{1}$

$$
\mathcal{M}_{\mathcal{N}=1} \longrightarrow \mathcal{M}_{\mathcal{N}=2}
$$

where the fibers of the map are the $\mathcal{N}=1$ "open string" vacua with fixed value of $\mathcal{N}=2$ "closed string" moduli. In a fixed charge sector, the extension is finite modulo continuous open string moduli, and is accompanied with a rich algebraic structure whose physical consequences are only beginning to emerge. The aspect emphasized in [1] is the action of the Galois group on the extending vacua, locally around large volume point. What we study in the present paper is how these local extensions fit together into the global structure of (1.1). This is a generalization of the work [2].

In the rest of the introduction, we describe the geometric (Hodge theoretic) situation, and then summarize our main results. The bulk of the paper is devoted to explicit calculations. We include a brief discussion section at the end, but the broader lessons for the landscape of $\mathcal{N}=1$ string vacua will be extracted elsewhere.

The geometric situation underlying our calculations involves, first of all, a smooth, quasi-projective family of Calabi-Yau threefolds $\mathcal{Y} \rightarrow B$, with semi-stable compactification $\overline{\mathcal{Y}} \rightarrow \bar{B}$. To keep that part simple, we will be working with the earliest list of four examples, one-parameter hypersurfaces in weighted projective space, originally studied in $[3,4]$. The list includes (the mirror manifolds of): the quintic $\mathbb{P}_{11111}^{4}[5]$, the sextic $\mathbb{P}_{11112}^{4}[6]$, the octic

\footnotetext{
${ }^{1}$ We are using a "mostly mathematical" notation throughout the paper, with occasional physics terminology when we do not know the correct mathematical equivalent. Equation (1.1) means that there exists a nice map from the $\mathcal{N}=1$ moduli space to the $\mathcal{N}=2$ moduli space, as we presently explain. As physicists, we would point the arrow in the direction of lower supersymmetry, as in, $\mathcal{N}=2 \rightarrow$ $\mathcal{N}=1$.
} 
$\mathbb{P}_{11114}^{4}[8]$ and the dectic $\mathbb{P}_{11125}^{4}[10]$. So, the base of our family will be a thricepunctured projective line, which we parameterize with a complex variable $z$ taking values $0,1, \infty$ at the three singular points: $B \cong \mathbb{P}^{1} \backslash\{0,1, \infty\}$.

The middle cohomology groups of the members of our family, $H^{3}\left(Y_{z}, \mathbb{C}\right)$, are four-dimensional symplectic vector spaces, and, as $z$ varies over $B$, fit together to a holomorphic vector bundle, $\mathcal{H}_{\mathbb{C}}$, that is naturally flat because the fibers contain the locally constant integral lattice $H^{3}\left(Y_{z}, \mathbb{Z}\right)$, fitting together to the local system $\mathcal{H}_{\mathbb{Z}}$. The global structure of the bundle $\mathcal{H}_{\mathbb{C}}=$ $\mathcal{H}_{\mathbb{Z}} \otimes \mathcal{O}_{B}$ is encoded in the monodromy representation

$$
\rho: \pi_{1}(B) \rightarrow \operatorname{Sp}(4, \mathbb{Z})
$$

In the usual conventions, the boundary point $z=z_{\mathrm{LV}}=0$ is the point of maximal unipotent monodromy (large volume point), $z=z_{\mathrm{C}}=1$ is the conifold point with unipotent monodromy of rank 1, while the Gepner point $z=z_{\mathrm{G}}=\infty$ has monodromy of finite order $(5,6,8$ and 10 in the four examples, respectively). We will imagine the base point implicit in (1.2) to be located close to $z_{\mathrm{LV}}$, and denote the corresponding fixed symplectic lattice by $\left(H_{\mathbb{Z}},\langle\cdot, \cdot\rangle\right)$. A choice of basis in $H_{\mathbb{Z}}$ allows to write matrices representing generators of $\pi_{1}(B)$, which we denote by $M_{\mathrm{LV}}, M_{\mathrm{C}}, M_{\mathrm{G}}$, and which satisfy

$$
M_{\mathrm{LV}} \cdot M_{\mathrm{C}}=M_{\mathrm{G}}
$$

A specific basis of $H_{\mathbb{Z}}$ is determined from the intrinsic properties of the variation of Hodge structure associated with $\mathcal{Y} \rightarrow B$, and its degeneration at $\bar{B} \backslash B$. From the fact that $N:=M_{\mathrm{LV}}-i d$ is nilpotent $\left(N^{4}=0\right)$ and maps integral vectors to integral vectors, the spaces $\operatorname{Im}\left(N^{3-j}\right) / \operatorname{Im}\left(N^{4-j}\right)$ for $j=$ $0,1,2,3$ are (projectively) rational. Using additional information from the symplectic form determines an integral basis $\left(\gamma_{0}, \gamma_{1}, \gamma_{2}, \gamma_{3}\right)$ of $H_{\mathbb{Z}}$ that is adapted to the monodromy weight filtration

$$
W_{2 j}=\operatorname{Ker} N^{j+1}, \quad j=0,1,2,3
$$

(namely $\gamma_{j}$ generates $W_{2 j} / W_{2 j-2}$ ), up to a lower-triangular symplectic transformation that is integral except for a single constant of integration, $\alpha$, which corresponds to an indeterminacy $\gamma_{3} \rightarrow \gamma_{3}+\alpha \gamma_{0}$, and can a priori take any (imaginary) value. These constants can be determined and hence a basis fully fixed by studying the behavior at the conifold locus. Namely, one imposes that $\gamma_{3}$ vanish at $z=z_{\mathrm{C}}$, and $M_{\mathrm{C}}$ send $\gamma_{0} \mapsto \gamma_{0}+\gamma_{3}$, and leave $\gamma_{1}, \gamma_{2}$, untouched. 
In practice, the task is accomplished by calculating the periods of the holomorphic three-form, viz., the restrictions

$$
\varpi_{j}=\left.\left\langle\gamma_{j}, \cdot\right\rangle\right|_{F^{3} \mathcal{H}}
$$

via the polarization $\langle\cdot, \cdot\rangle$, to the first step of the Hodge filtration $F^{*} \mathcal{H}$ on $\mathcal{H}_{\mathbb{C}}$. More precisely, choosing a non-zero section $\Omega \in \Gamma\left(B, F^{3} \mathcal{H}\right)$ determines a Picard-Fuchs differential equation satisfied by any (complex) period

$$
\mathcal{L} \varpi(\Omega)=0
$$

and studying the analytic properties of the solutions of that differential equation provides all the data listed above. The first such calculation was completed for the quintic in [5], with further explanations in [6,7]. For more recent discussions, see, e.g., [8] which in particular points out a severe ambiguity of this procedure or [9].

Once this is done, the (conjecturally) irrational constant $\alpha$ mentioned above features as an entry of the limiting period matrix with respect to the canonical mirror map coordinate

$$
q=\left.\exp 2 \pi i \frac{\left\langle\gamma_{1}, \cdot\right\rangle}{\left\langle\gamma_{0}, \cdot\right\rangle}\right|_{\left(F^{3} \mathcal{H}\right)^{\times}} .
$$

The fact that in general

$$
\alpha \in \frac{\zeta(3)}{(2 \pi i)^{3}} \mathbb{Q}
$$

is explained by the physics origin of this constant (in perturbative corrections to the sigma-model on the A-model manifold), as well as by motivation [9]. It also meshes nicely with the recent discussions of integral structures on quantum cohomology in the context of the gamma genus, see [10-12].

We now introduce the main complication, which models extension by D-branes (1.1). It is the same as in several previous works [13-15]. For each fixed member of our family of Calabi-Yau threefolds, we find holomorphic curves $C_{z, k} \subset Y_{z}$ that vary generically locally uniquely (as algebraic cycles modulo algebraic equivalence) with $z$. Here, $k$ is an index running over a certain finite set $A$. Consideration of such a finite collection of curves is necessary because any given $C_{z, k}$ will, under continuous global variation of $z$, branch at specific locations in $B$, i.e., the local variation will not be unique, and the curve will not return to itself when the variation encircles 
those branch points. In other words, in order for the collection of curves to fit together into a globally well-defined algebraic cycle $\mathcal{C}$, we first have to extend the moduli space to an $|A|$-fold branched covering $\hat{B} \rightarrow B$. Schematically,

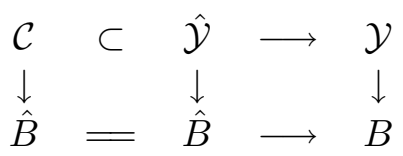

and the $C_{z, k}$ are components of the fibers of $\mathcal{C} \rightarrow B$. We assume that for fixed $z$, the $C_{z, k}$ for different $k$ are homologous to each other, and generically irreducible. We will call the branch locus of $\hat{B} \rightarrow B$ the "open string discriminant," and denote it by $D$. In the examples, $D$ is a finite number of points. (To be sure, the extension (1.9) extends to the compactification $\overline{\mathcal{Y}} \rightarrow \bar{B}$, and $\hat{\bar{B}} \rightarrow \bar{B}$ can also be branched at $\bar{B} \backslash B$. This plays an important role in our analysis. But when we speak of open string discriminant, we only mean points that were not on the boundary before.)

Associated to the algebraic cycle $\mathcal{C} \rightarrow B$, we have a variation of mixed Hodge structure. Locally on $B$, the extension is encoded in the Abel-Jacobi map to the intermediate Jacobian

$$
\mathcal{J}=F^{2} \mathcal{H} \backslash \mathcal{H}_{\mathbb{C}} / \mathcal{H}_{\mathbb{Z}}
$$

as discussed extensively in the literature, loc. cit.. Specifically, to a local family of homologically trivial cycles, such as $C_{z}=C_{z, k}-C_{z, k^{\prime}}$ in some simply connected open set in $B$, we can associate a normal function, $\nu$, as a holomorphic section of $\mathcal{J}$ satisfying Griffiths transversality

$$
\nabla \tilde{\nu} \in F^{1} \mathcal{H} \otimes \Omega_{B}
$$

Here $\tilde{\nu}$ is a lift of $\nu$ to $\mathcal{H}_{\mathbb{C}}$ and $\nabla$ is the Gauss-Manin connection. Such a lift can be conveniently represented by an integral over a three-chain bounding $C_{z}$. Actually, as a consequence of (1.11) (and surjectivity of the infinitesimal period mapping), the complete information about the extension class $\nu$ can be recovered from the inhomogeneous Picard-Fuchs equation

$$
\mathcal{L} \tau(\Omega)=f
$$

satisfied by the truncated normal function

$$
\tau=\left.\langle\tilde{\nu}, \cdot\rangle\right|_{F^{3} \mathcal{H}}
$$


(cf. (1.5)). The basic idea behind this statement is that in the Hodge decomposition of $\tilde{\nu}$, the truncated normal function gives the $(0,3)$ part, and the first derivatives of $\tau$ give the $(1,2)$ part. Moreover, as emphasized in [15], the inhomogeneity on the right-hand side of (1.12) is local and additive in the boundary cycle $C_{z}$. Since the integral (period) ambiguity of $\tau$ drops out of the differential equation (1.12), this means that we can associate an inhomogeneity, $f_{k}$, to each curve, $C_{z, k}$, by itself, such that when $C_{z}=C_{z, k}-C_{z, k^{\prime}}$, we have

$$
f=f_{k}-f_{k^{\prime}}
$$

More formally, and for the global issues which we propose to study in the present paper, it is convenient to fix a "marking" on $A$, the finite set labeling the $C_{z, k}$. The simplest way to do this is to include a locally constant (technically, of vanishing infinitesimal invariant) and globally invariant curve in the same homology class that serves as reference point for the chain integrals. Namely, if we label this reference curve with $k=0$, then the point of is that $f_{0}=0$, and we might as well identify $f_{k}$ with the inhomogeneity associated to $C_{z, k}-C_{z, 0}$, which is more canonical. In the calculations, this additional cycle will often be implicit, though we promise to display it at least once (see Equation (2.9)).

Physically, in an $\mathcal{N}=1$ compactification of the type II/I superstring, the truncated normal function $\tau$ gives the contribution to the space-time superpotential $\mathcal{W}$ for the chiral scalar fields coming from $\mathcal{N}=2$ vector-multiplets, that is made by a D-brane configuration whose algebraic characteristic class is the cycle under consideration, after integrating out all (massive) degrees of freedom on the D-brane worldvolume.

To state the main results of our calculations, we denote by $\mathcal{A}$ the local system over $B$ obtained by tensoring the data of the extension $\hat{B} \rightarrow B$ with $\mathbb{Z}$. Continuation of the bounding chains over $B$ then really is an extension of local systems

$$
\mathcal{H}_{\mathbb{Z}} \longrightarrow \hat{\mathcal{H}}_{\mathbb{Z}} \longrightarrow \mathcal{A}
$$

that underlies the variation of mixed Hodge structure, and which we recover from the solutions of the inhomogeneous Picard-Fuchs equation. In other words, we will determine the monodromy representation

$$
\hat{\rho}: \pi_{1}(B \backslash D) \rightarrow S_{A} \times i \operatorname{Sp}(4, \mathbb{Z}),
$$


where $S_{A}$ is the symmetric group and

$$
i \operatorname{Sp}(4, \mathbb{Z})=\left(H_{\mathbb{Z}}\right)^{A} \ltimes \operatorname{Sp}(4, \mathbb{Z})
$$

The factor $\left(H_{\mathbb{Z}}\right)^{A}$ arises because the bounding chains will only return up to closed three-cycles, and manifests itself in shifts of the truncated normal function by solutions of the homogeneous equation. The crux of the computation is that these shifts are indeed integral periods.

We find that, in analogy with the homogeneous case reviewed above, the integrality of monodromy can be determined by combining data from the large volume point and the conifold. This data can be interpreted in terms of limiting values of normal functions studied in full generality in the work of Green-Griffiths-Kerr [16]. In a degeneration of maximal unipotent monodromy, the relevant statement for us is that with respect to the monodromy weight filtration (1.4), the lift of the normal function is integral modulo $W_{1}$, and rational modulo $W_{0}$. (It is integral modulo $W_{0}$ when the covering $\hat{\bar{B}} \rightarrow \bar{B}$ is trivial at $z_{\mathrm{LV}}$.) Moreover, the coefficient $a_{k}$ of the fundamental period $\varpi_{0}$ in the truncated normal function $\tau_{k}$ (in the limit $z \rightarrow z_{\mathrm{LV}}$, with respect to the canonical coordinates (1.7)) has an interpretation in terms of the geometry of the singular fiber $Y_{z_{\mathrm{LV}}}$, which leads to general expectations about the range of values analogous to (1.8). In our examples, we find that this coefficient is completely determined from the conifold monodromy, and our numerical results are consistent with the general expectations.

An interesting observation is that, at least in all examples that we study, the integral structure at $z_{\mathrm{LV}}$ (and in particular, the constants $a_{k}$ ) can also be determined by tracking the vanishing normal function to the open string discriminant, $D$, and imposing appropriate boundary conditions over there. We remark that this possibility is not a priori obvious (at least to us) because it requires a certain relation between the branch structure at $z_{\mathrm{LV}}$ and the number of components of $D$. We will emphasize this aspect in the discussion. As a practical matter, however, the coincidence is quite welcome because it over-constrains integrality of monodromy.

Here is an overview over the remainder of the paper:

We will start in Section 2 by identifying interesting cycles $\mathcal{C}$ in each of our four families of Calabi-Yau hypersurfaces. As in [1], we organize the search by looking for curves of low degree, and lines specifically. Drawing on the strategy employed by van Geemen [17-19], imposing certain discrete symmetries allows us to fully solve the problem in certain cases. We note that some of our lines actually belong to families (in the sense that they allow 
additional continuous deformations for fixed $z$ ), but we do not complete the analog of the discussion of van Geemen lines in $[18,20]$. Referring the interested reader to $[13,15]$ for the details of the method, and to appendix for a few intermediate steps in one example, we present the result of the calculation of the inhomogeneity $f_{k}$ for each of our cycles.

In Section 3, we localize our cycles to the large volume point $z_{\mathrm{LV}}$. Following [1], we perform a Newton-Puiseux expansion that separates the curves by residue field. We then check that the A-model expansion of the truncated normal function (the space-time superpotential) satisfies the "D-logarithm integrality" discovered in [1], and recently proven in [21]. All the new cycles from Section 2 turn out to have residue fields that are abelian extension of $\mathbb{Q}$. Therefore, in order to have a more complete set of examples for the monodromy calculations, we also include the (non-abelian) conics from [1].

Section 4 then is concerned with the main calculations. For each of the cycles, we expand periods and truncated normal functions at the conifold and at the open string discriminant. Numerical analytic continuation along certain paths in $B$ determines the relevant change of basis and monodromy matrices. The one friendly aspect is that all components of the open string discriminants are on the real axis.

We summarize our numerical results in Section 5, see in particular table 1 , and discuss the arithmetic significance to the best of our abilities.

Note added: The referees' comments prompt us to translate the sentence below (1.1) into a more mathematical language. The phrase "fixed charge sector" means that we are considering algebraic curves with a fixed degree (in particular, we are considering lines and conics). "Continuous open string moduli" are (unobstructed) deformations of the cycles for fixed $z$, or algebraic equivalences. Such deformations do not affect the inhomogeneous Picard-Fuchs equation or the normal function. In other words, we are exploiting that the Griffiths group of algebraic cycles modulo algebraic equivalence is filtered by the degree of a representative cycle, and this filtration is finite at each step. Based on this, our calculations should be useful input for more rigorous discussions of algebraic cycle groups and their AbelJacobi image.

\section{From curves to residues}

For completeness, we begin with some of the standard homogeneous data. Our Calabi-Yau manifolds are hypersurfaces of degree $d=5,6,8,10$ in 
weighted projective space

$$
\{W=0\} \subset \mathbb{P}_{w_{1}, w_{2}, w_{3}, w_{4}, w_{5}}^{4},
$$

where $\left(w_{1}, w_{2}, w_{3}, w_{4}, w_{5}\right)=(1,1,1,1,1),(1,1,1,1,2),(1,1,1,1,4),(1,1,1$, $2,5)$ are the weights, and $d=\sum_{i} w_{i}$. The Fermat-polyhedron-Dwork pencil from which we construct the mirror manifold is specified by the family of polynomials

$$
W=\sum_{i} \frac{w_{i}}{d} x_{i}^{d / w_{i}}-\psi \prod_{i} x_{i}
$$

where the global complex structure parameter is related to $\psi$ via

$$
z=\psi^{-d}
$$

The convenient normalization of the holomorphic three-form is

$$
\Omega=\frac{|G|}{(2 \pi i)^{3}} \operatorname{Res}_{W=0} \frac{\psi \omega}{W},
$$

where $\omega=\alpha(v), \alpha=d x_{1} \wedge \cdots \wedge d x_{5}, v=\sum w_{i} x_{i} \partial_{i}$ and $|G|=d^{3} / \prod w_{i}$ is the order of the Greene-Plesser group. The three-form satisfies the PicardFuchs equation

$$
\mathcal{L} \Omega=d \beta
$$

where $\beta$ is a certain two-form and $d=d_{\text {rel }}$ is the fiberwise exterior derivative. The Picard-Fuchs operator can be written as

$$
\mathcal{L}=\theta^{4}-z\left(\theta+r_{1}\right)\left(\theta+r_{2}\right)\left(\theta+r_{3}\right)\left(\theta+r_{4}\right),
$$

where $\quad\left(r_{1}, r_{2}, r_{3}, r_{4}\right)=\left(\frac{1}{5}, \frac{2}{5}, \frac{3}{5}, \frac{4}{5}\right),\left(\frac{1}{6}, \frac{2}{6}, \frac{4}{6}, \frac{5}{6}\right),\left(\frac{1}{8}, \frac{3}{8}, \frac{5}{8}, \frac{7}{8}\right),\left(\frac{1}{10}, \frac{3}{10}, \frac{7}{10}, \frac{9}{10}\right)$ are the "indices at infinity," and $\theta \equiv z \partial_{z}$.

\subsection{Quintic}

The generic quintic threefold contains 2875 lines. That number not being divisible by 3 , while the Dwork pencil is invariant under cyclic permutation of $\left(x_{1}, x_{2}, x_{3}\right)$, suggests that there should exist $\mathbb{Z} / 3$-invariant lines for generic values of $\psi$. It is not very hard to see that there are, up to $\mathrm{SL}(2, \mathbb{C})$ transformations on homogeneous coordinates $(u, v)$, and conjugacy class of cyclic 
permutation, precisely two different $\mathbb{Z} / 3$-equivariant parameterizations that are distinguished by whether the determinant of the generator acting on $(u, v)$ is 1 or a non-trivial cube root of unity, $\omega$. (Note that this distinction arises after fixing a particular linearization of the $\mathbb{Z} / 3$ action on projective space. Twisting the action with multiplication of the homogeneous coordinates by cube roots of unity maps the two possibilities onto each other.) We will need the first of those options later, while for the quintic we are left with the general ansatz

$$
x_{1}=u+v, \quad x_{2}=u+\omega v, \quad x_{3}=u+\omega^{2} v, \quad x_{4}=a u, \quad x_{5}=b u
$$

where $a, b$ are two parameters that are constrained by the condition that (2.7) be contained in the mirror quintic

$$
a b \psi=6, \quad a^{5}+b^{5}=27 .
$$

The solutions to these equations (and their images under symmetries of the quintic) yield the van Geemen lines. The original interest of these lines [17] was that they allow continuous (unobstructed) deformations for fixed $\psi$. The global structure of the corresponding families was worked out in [18], see also [20]. One of the results of this analysis is that the only other lines besides those contained in these families are the coordinate lines, such as

$$
C_{0}=\left\{x_{1}+x_{2}=0, x_{3}+x_{4}=0, x_{5}=0\right\} .
$$

It follows from elementary considerations (or the explicit calculations in [13]) that these coordinate lines have a vanishing inhomogeneity, i.e.,

$$
\int_{C_{0}} \beta=0,
$$

where $\beta$ is the two-form in (2.5). This vanishing reflects the fact that the Abel-Jacobi image of the coordinate lines is torsion. ${ }^{2}$ Since they are in addition of primitive degree, the $C_{0}$ (and their integral multiples) are ideally suited to serve as reference cycle for the monodromy calculations as mentioned in the introduction. All our other examples have similar coordinate lines.

\footnotetext{
${ }^{2}$ For the coordinate lines, this follows from elementary symmetry considerations. It is however not clear whether this statement follows from known results about algebraic cycles on Calabi-Yau threefolds, whose general versions would be widely open. We thank the referee for instructive comments on this point.
} 
On the other hand, the van Geemen lines have a non-trivial Abel-Jacobi image. This was pointed out via an infinitesimal calculation in [18], while the complete inhomogeneity was determined in [1] to be

$$
f_{\omega}(z)=\frac{1+2 \omega}{(2 \pi i)^{2}} \cdot \frac{32}{45} \cdot \frac{\frac{63}{\psi^{5}}+\frac{1824}{\psi^{10}}-\frac{512}{\psi^{15}}}{\left(1-\frac{128}{3 \psi^{5}}\right)^{5 / 2}}
$$

The factor of $1-\frac{128}{3 \psi^{5}}$ in the denominator is the discriminant of the equations (2.8), when $a^{5}=b^{5}$. It is worthwhile pointing out that when $a=b$ in (2.7), mapping $\omega \mapsto \omega^{2}$ can be compensated by $x_{2} \leftrightarrow x_{3}, x_{4} \leftrightarrow x_{5}$, which leaves the holomorphic three-form invariant. This explains in elementary terms why changing the sign of the square-root in (2.11) is equivalent to changing $\omega \mapsto \omega^{2}$. More abstractly, we might say that the cycle generated by the van Geemen lines modulo algebraic equivalence provides a twofold cover of $\psi$-space (the base of our family of varieties) branched at $\psi=0$ and $3 \psi^{5}=128$. In particular, this extension does not split globally over $\mathbb{Q}(\omega)$.

We now briefly review some conics on the mirror quintic found in [1]. Consider the $\mathbb{Z}_{2} \times \mathbb{Z}_{2}$-invariant ansatz

$$
\begin{aligned}
C_{a, b}=\{ & x_{1}+x_{3}+a x_{5}, x_{2}+x_{4}+a x_{5}, x_{3}^{2}+x_{4}^{2}+b x_{3} x_{4} \\
& \left.+\left(a+\frac{1}{2} a b\right)\left(x_{3}+x_{4}\right) x_{5}+\frac{1}{8}\left(-\psi a+6 a^{2}+2 a^{2} b\right) x_{5}^{2}\right\} .
\end{aligned}
$$

These conics lie on the quintic precisely if

$$
\begin{aligned}
64+5 a^{3} \psi^{2}-40 a^{4} \psi+12 a^{5} & =0 \\
\psi-2 a+a b^{2} & =0 .
\end{aligned}
$$

These are, for fixed generic $\psi$, ten different conics, so the covering $\hat{B} \rightarrow$ $B$ is quite a bit more interesting than for the van Geemen lines. Before discussing it, we note that passing to the global coordinate $z=\psi^{-5}$ is easily accomplished since Equations (2.13) are invariant under $(\psi, a) \rightarrow(\eta \psi, \eta a)$, when $\eta^{5}=1$.

Now, the nature of the symmetry $b \rightarrow-b$ shows that the ten conics group as pairs of conics in five different planes determined by the first equation of (2.13). That symmetry acts trivially when $a=\psi / 2$, which under the first equation can be seen to coincide with the discriminant locus of the van Geemen lines, $3 \psi^{5}=128$. Indeed, at this point, the conics (2.12) are reducible to two members of the van Geemen family. One may check that the conics are also reducible at $7 \psi^{5}=128$, but this is not a branch point of the covering (2.13). 
The discriminant of the first equation in (2.13) is

$$
-5308416+26104832 \psi^{5}+459 \psi^{10}=0 .
$$

In $B$ (parameterized by $z=\psi^{-5}$ ), these are the two points

$$
z_{ \pm}=\frac{50986 \pm 6875 \sqrt{55}}{20736} .
$$

The inhomogeneity corresponding to $C_{a, b}$ was also calculated in [1]. The result can be simplified to

$$
\begin{aligned}
f_{a, b}= & \frac{1}{\pi^{2}} \cdot \frac{b}{8640(2 a-\psi)^{5}\left(12 a^{2}-32 a \psi+3 \psi^{2}\right)^{5}} \\
& \times\left(-366917713920-1016582897664 a^{4} \psi+3474322882560 a^{3} \psi^{2}\right. \\
& -3601465344000 a^{2} \psi^{3}+2232487772160 a \psi^{4}+1993006776320 \psi^{5} \\
& -1127509778432 a^{4} \psi^{6}-62141296640 a^{3} \psi^{7}+139109736960 a^{2} \psi^{8} \\
& -48377468160 a \psi^{9}+8404041600 \psi^{10}+92770596 a^{4} \psi^{11} \\
& \left.-308068920 a^{3} \psi^{12}+34766415 a^{2} \psi^{13}+486000 a \psi^{14}\right) .
\end{aligned}
$$

Note that the denominator of (2.16) vanishes when $2 a=\psi$, which implies $3 \psi^{5}=128$ as we have seen above, or when $12 a^{2}-32 a \psi+3 \psi^{2}=0$, which implies (2.14). Thus, as it should be, the three components of the open string discriminant

$$
z_{\mathrm{D}_{11}}=z_{-}, \quad z_{\mathrm{D}_{12}}=z_{+}, \quad z_{\mathrm{D}_{2}}=\frac{3}{128}
$$

are manifest in the denominator of the inhomogeneity.

\subsection{Sextic}

This subsection contains the first new results. As is well known, the generic number of lines on a weighted sextic Calabi-Yau threefold is $7884 .{ }^{3}$ Since this is divisible by 3 , it is possible for the cyclic permutations of the homogeneous variables, such as $\left(x_{1}, x_{2}, x_{3}\right) \rightarrow\left(x_{3}, x_{1}, x_{2}\right)$ to act freely on the set of lines.

\footnotetext{
${ }^{3}$ The mirror formula for the number of lines is $\frac{d}{\prod w_{i}}\left[\frac{d^{d}}{\prod w_{i}^{w_{i}}}-\frac{d !}{\prod w_{i} !}\left(3 d H_{d}-\right.\right.$ $\left.\left.3 \sum w_{i} H_{w_{i}}+2\right)\right]$.
} 
Working out the equations, we find that indeed there are no $\mathbb{Z}_{3}$-invariant lines on (the one-parameter family mirror to) $\mathbb{P}_{11112}^{4}[6]$.

As an example that divisibility (of the generic number of solutions by the order of a symmetry group) does not imply absence of solutions (invariant under that symmetry), we consider lines invariant under the $\mathbb{Z}_{2}$ symmetry

$$
\left(x_{1}, x_{2}, x_{3}, x_{4}, x_{5}\right) \mapsto\left(x_{2}, x_{1}, x_{4}, x_{3}, x_{5}\right)
$$

With a parameterization ansatz

$$
\begin{aligned}
& x_{1}=a_{1} u+v, \quad x_{2}=a_{1} u-v, \\
& x_{3}=u+a_{2} v, \quad x_{4}=u-a_{2} v, \\
& x_{5}=a_{3} u^{2}+a_{4} v^{2},
\end{aligned}
$$

we find the space of such lines factors over $\mathbb{Q}$ into several components. The simplest of those has $a_{1}$ and $a_{2}$ equal to sixth roots of -1 , and $a_{3}=a_{4}=$ $\sqrt{-3 \psi}$. These are nothing but the curves studied in [14]. The corresponding inhomogeneity was found to be proportional to $z^{1 / 2}$, and the monodromy of solutions was also completely worked out. In the present paper, we do not wish to discuss these "toric" curves any further.

The next more complicated lines with $\mathbb{Z}_{2}$ symmetry (2.18) turn out to also be invariant under a second $\mathbb{Z}_{2}$ acting as

$$
\left(x_{1}, x_{2}, x_{3}, x_{4}, x_{5}\right) \mapsto\left(x_{3}, x_{4}, x_{1}, x_{2}, x_{5}\right)
$$

Imposing (2.18) and (2.20) forces the parameters of our ansatz to respect

$$
a_{2}=a_{1}, \quad a_{4}=a_{3}
$$

Eliminating $a_{4}$ (and ignoring the toric solutions), the equations boil down to

$$
\begin{gathered}
1-18 a_{2}^{2}+111 a_{2}^{4}-252 a_{2}^{6}+111 a_{2}^{8}-18 a_{2}^{10}+a_{2}^{12}+\psi^{3} \\
+15 a_{2}^{4} \psi^{3}+32 a_{2}^{6} \psi^{3}+15 a_{2}^{8} \psi^{3}+a_{2}^{12} \psi^{3}=0
\end{gathered}
$$

and its images under multiplying $a_{2}$ by a third root of unity.

With these out of the way, we can complete the reduction of the curves with only a single $\mathbb{Z}_{2}$ symmetry, (2.18). There are two more components 
over $\mathbb{Q}$. The first is characterized by the vanishing of

$$
1+2 a_{2}^{6}+a_{2}^{12}-4 a_{2}^{6} \psi^{3}
$$

while the second by

$$
\begin{aligned}
& 1953125+7812500 a_{2}^{6}+11718750 a_{2}^{12}+7812500 a_{2}^{18}+1953125 a_{2}^{24} \\
& \quad+2062500 \psi^{3}-6250000 a_{2}^{6} \psi^{3}-16625000 a_{2}^{12} \psi^{3}-6250000 a_{2}^{18} \psi^{3} \\
& \quad+2062500 a_{2}^{24} \psi^{3}+726000 \psi^{6}-3156000 a_{2}^{6} \psi^{6}+12236000 a_{2}^{12} \psi^{6} \\
& \quad-3156000 a_{2}^{18} \psi^{6}+726000 a_{2}^{24} \psi^{6}+85184 \psi^{9}-484864 a_{2}^{6} \psi^{9} \\
& \quad-180096 a_{2}^{12} \psi^{9}-484864 a_{2}^{18} \psi^{9}+85184 a_{2}^{24} \psi^{9}+18944 a_{2}^{6} \psi^{12} \\
& \quad+18688 a_{2}^{12} \psi^{12}+18944 a_{2}^{18} \psi^{12}-1024 a_{2}^{12} \psi^{15}
\end{aligned}
$$

We have calculated the inhomogeneity corresponding to (2.23), and found it to vanish. We suspect the same to hold for (2.22), although we have not completed the calculation. (The basis for this conjecture is that the discriminant meets the conifold locus $\psi=1$.) We have also not calculated the inhomogeneity corresponding to (2.24).

Since these results do not yield any new inhomogeneity for the PicardFuchs equation of the sextic, we will drop it from the list for the rest of this paper.

\subsection{Octic}

The number of lines on the weighted octic Calabi-Yau threefold is 29504. This is not divisible by 3, so there should be analogs of the van Geemen lines. Indeed, let us parameterize lines invariant under

$$
\left(x_{1}, x_{2}, x_{3}, x_{4}, x_{5}\right) \mapsto\left(x_{2}, x_{3}, x_{1}, x_{4}, x_{5}\right)
$$

via

$x_{1}=u+v, \quad x_{2}=u+\omega v, \quad x_{3}=u+\omega^{2} v, \quad x_{4}=a u, \quad x_{5}=b u^{4}+c u v^{3}$,

where $\omega$ is a non-trivial cube root of unity. We find that the space of such lines factors globally in several components. The first of those has

$$
a^{8}=3^{4}
$$


while the second

$$
a^{2} \psi^{2}=21
$$

Note that both of these expressions are invariant under $(\psi, a) \rightarrow\left(\eta \psi, \eta^{-1} a\right)$ when $\eta^{8}=1$, so that the corresponding cycle $\mathcal{C}$ is indeed well-defined over $B$.

We have calculated the corresponding inhomogeneities, with the following results. For the first component, (2.27), we find

$$
f_{1}(z)=\frac{\sqrt{-3}}{(2 \pi i)^{2}} \cdot \frac{3}{16} \cdot \frac{\psi\left(8+\psi^{2}\right)}{\left(\psi^{2}-7\right)^{5 / 2}} .
$$

For the convenience of the reader, we explain a few of the intermediate steps leading to (2.29) in the appendix.

The second component of lines on the octic with the $\mathbb{Z}_{3}$ symmetry (2.25) gives inhomogeneity

$$
f_{2}(z)=\frac{\sqrt{-7}}{(2 \pi i)^{2}} \cdot \frac{147}{16} \cdot \frac{-823543+184534 \psi^{8}+129 \psi^{16}}{\psi^{4}\left(\psi^{8}-2401\right)^{5 / 2}} .
$$

Finally, we note that we have also studied lines with only a $\mathbb{Z}_{2}$ symmetry exchanging two coordinates. For all the ones for which we have computed the inhomogeneity, it vanishes.

\subsection{Dectic}

Last on the list of one-parameter Calabi-Yau hypersurfaces is the weighted dectic, $\mathbb{P}_{11125}^{4}[10]$. It contains generically 231200 lines, a number also not divisible by 3 . Searching for lines that are invariant under cyclic permutation of the first three variables, we find that the ansatz analogous to (2.7) allows only three parameters (the coefficient of $u^{2}$ in $x_{4}$ and the coefficients of $u^{5}$, $u^{2} v^{3}$ in $x_{5}$ ), constrained by a total of four independent equations (the coefficients of $u^{10}, u^{7} v^{3}, u^{4} v^{6}, u v^{9}$ in $W$ ), so there are generically no solutions. This is where we remember the other possible $\mathbb{Z}_{3}$-equivariant parameterization. The ansatz

$$
\begin{aligned}
& x_{1}=u+v, \quad x_{2}=\omega u+\omega^{2} v, \quad x_{3}=\omega^{2} u+\omega v, \quad x_{4}=a u v \\
& x_{5}=b u^{4} v+c u v^{4}
\end{aligned}
$$

yields three parameters constrained by three equations (coefficients of $u^{2} v^{8}$, $\left.u^{5} v^{5}, u^{8} v^{2}\right)$. We find that there are two components, characterized by the 
vanishing of $a^{5}+3^{5}$ and $513+a^{5}-10 a^{2} \psi^{2}$, respectively. It turns out that the first has vanishing inhomogeneity, while the second gives

$$
\begin{aligned}
f(z)= & \frac{1+2 \omega}{(2 \pi i)^{2}} \cdot \frac{9}{50 \psi^{5}(b-a \psi)^{5}\left(a^{3}-4 \psi^{2}\right)^{5}} \cdot \\
& \left(87483691656-9805676940 a^{2} \psi^{2}+402856335 a^{4} \psi^{4}-2843845767 a \psi^{6}\right. \\
& +448788924 a^{3} \psi^{8}+10768937688 \psi^{10}-321417648 a^{2} \psi^{12} \\
& \left.+810896 a^{4} \psi^{14}-15348960 a \psi^{16}+299200 a^{3} \psi^{18}\right),
\end{aligned}
$$

where the parameters $a, b, c, \omega$ in the ansatz (2.31) are determined by the system of equations

$$
\begin{aligned}
513+a^{5}-10 a^{2} \psi^{2} & =0 \\
27+b^{2}-2 a b \psi & =0
\end{aligned}
$$

as well as $c=2 a \psi-b, \omega$ : a non-trivial cube root of unity. This system is somewhat similar to Equations (2.13) for conics on the quintic, in that there are five pairs of solutions for fixed $\psi$. The open string discriminant has two components

$$
z_{\mathrm{D}_{1}}=\frac{128}{3^{6} \cdot 19^{3}}, \quad z_{\mathrm{D}_{2}}=\frac{1}{243}
$$

also apparent in $(2.32)$ (recall that in present conventions, $z=\psi^{-10}$ ).

\section{Algebraic integrality}

Consider the A-model expansion of the truncated normal function $\tau$ (1.13) associated to an algebraic cycle, around the point of maximal unipotent monodromy, $z=z_{\mathrm{LV}}=0$. It is defined as the expansion in the mirror variable $q$ from Equation (1.7), of the quantity

$$
\mathcal{W}_{A}=\left.\frac{\langle\tilde{\nu}, \cdot\rangle}{\left\langle\gamma_{0}, \cdot\right\rangle}\right|_{\left(F^{3} \mathcal{H}\right)^{\times}} .
$$

On general grounds, explained in the introduction, the A-model expansion takes the form

$$
\mathcal{W}_{A}=\frac{s}{2 \pi i r} \log q+a+\frac{1}{(2 \pi i)^{2}} \sum_{d=1}^{\infty} \tilde{n}_{d} q^{d / r}
$$


Here, $r$ and $s$ are integers, with $r$ measuring the ramification index of the cycle at $z=z_{\mathrm{LV}}($ and $s$ is defined $\bmod r$ ). Namely, the cycle is really defined over the locally extended moduli space with local coordinate $z^{1 / r}$. Moreover, $a$ is an a priori arbitrary complex constant.

We emphasize again that the "classical terms" in (3.2) (the constant $a$ and the $\log q$ term) are not determined by the inhomogeneous Picard-Fuchs equation that we calculated in the previous section. This follows from the definitions on account of the fact that $\varpi_{0}$ and $\varpi_{1}$ (Equation (1.5)) are periods, i.e., solutions of the homogeneous Picard-Fuchs equation. Instead, the classical terms can be recovered from a monodromy calculation, as we will do in the next section. In this section, we will concentrate on the non-trivial part of the $q$-expansion in (3.2) (the "instanton expansion"). This serves two purposes. First, we want to explain the splitting of the extension $\hat{B} \rightarrow B$ at $z_{\mathrm{LV}}$, and emphasize again that the coefficients $\tilde{n}_{d}$ are not in general rational numbers. Second, we want to display the algebraic integrality discussed in $[1,21]$ that is nevertheless inherent in the instanton expansion.

\subsection{Octic}

The instanton expansion we find for our lines on the octic is rather similar to that of the van Geemen lines originally studied in [1]. In particular, the field extension is quadratic and appears only as an overall constant.

3.1.1. First component. Solving the differential equation with $f_{1}$ from (2.29) as inhomogeneity, and doing the expansion, we find, modulo classical terms

$$
\begin{aligned}
\frac{(2 \pi i)^{2}}{\sqrt{-3}} \cdot \mathcal{W}_{A} \equiv & 768 q^{1 / 4}+19584 q^{1 / 2}+860160 q^{3 / 4}+48733440 q \\
& +\frac{79882616832}{25} q^{5 / 4}+230232655872 q^{3 / 2}+\frac{868448460865536}{49} q^{7 / 4} \\
& +1432733965743360 q^{2}+120259506663856128 q^{9 / 4} \\
& +\frac{259997807371266134016}{25} q^{5 / 2}+\frac{111494235354933550841856}{121} q^{11 / 4} \\
& +83296525620921045651456 q^{3}+\cdots
\end{aligned}
$$

Note that while the field extension is quadratic, the good local variable is in fact $q^{1 / 4}$ (i.e., $r=4$ ). Taking account of the sign of the square-root of -3 , this corresponds to a degree 8 covering, locally organized in two groups of degree 4. This can in fact also be seen from the defining Equations (2.26) and (2.27). Multiplying $a$ with an eighth root of unity in general will give 
a different curve (the calculation in the appendix was done for $a^{2}=3$ ), but $(a, \omega) \rightarrow\left(-a, \omega^{2}\right)$ can be compensated by $\left(x_{2}, x_{3}, x_{4}\right) \rightarrow\left(x_{3}, x_{2},-x_{4}\right)$, an operation that leaves the holomorphic three-form invariant.

Since $\sqrt{-3}$ appears only as an overall constant, the expected integrality takes a fairly simple form. It can be written by twisting the standard OoguriVafa multi-cover formula by the corresponding quadratic residue character. Namely, with

$$
\sum_{d} \tilde{n}_{d} q^{d / 4}=\sum_{d, k} n_{d} \frac{\left(\frac{-3}{k}\right)}{k^{2}} q^{d k / 4}
$$

where $\left(\frac{-3}{k}\right)=0,1,-1$ if $k=0,1,2 \bmod 3$, the $n_{d}$ are integers (times $\left.\sqrt{-3}\right)$. For instance

$$
\begin{aligned}
n_{7} & =\tilde{n}_{7}-\frac{\tilde{n}_{1}}{49}=\sqrt{-3} \cdot 17723437976832, \\
n_{11} & =\tilde{n}_{11}+\frac{\tilde{n}_{1}}{121}=\sqrt{-3} \cdot 921439961611021081344
\end{aligned}
$$

3.1.2. Second component. This case has $r=1$, and a quadratic extension $\mathbb{Q}(\sqrt{-7})$ as residue field. There is no branching at $z_{\mathrm{LV}}$. The first few terms of the A-model expansion are

$$
\begin{aligned}
\frac{(2 \pi i)^{2}}{\sqrt{-7}} \mathcal{W}_{A} \equiv & 77672448 q+2364921695023104 q^{2} \\
& +139205158983427963682816 q^{3} \\
& +10833679402194213394854742437888 q^{4} \\
& +\frac{24618206559572019809666493201002121265152}{25} q^{5}+\cdots
\end{aligned}
$$

$\operatorname{In}$

$$
\sum_{d} \tilde{n}_{d} q^{d}=\sum_{d, k} n_{d} \frac{\left(\frac{-7}{k}\right)}{k^{2}} q^{k d}
$$

the $n_{d}$ are integral,e.g.,

(3.8) $n_{5}=\tilde{n}_{5}+\frac{\tilde{n}_{1}}{25}=\sqrt{-7} \cdot 984728262382880792386659728040087957504$.

\subsection{Dectic}

We now turn to the extension (2.33) of the dectic moduli space, with corresponding inhomogeneity (2.32). Because of the high degree of these equation, 
we cannot solve them in terms of radicals as we did before. Rather, we rely on expanding the parameters around $\psi^{-10}=z=z_{\mathrm{LV}}=0$ in a fractional power series.

Consider first the equation for $a$. The upper boundary of the Newton polygon of the first of (2.33) consists of two segments, one of slope 1 and one of slope $-2 / 3$. The corresponding local field extensions can be read off from the coefficients on those segments. We see that the power series for $a$ has coefficients in $\mathbb{Q}(\sqrt{513 / 10})$ and $\mathbb{Q}\left(10^{1 / 3}\right)$, respectively.

We can now insert these solutions into the second of Equations (2.33). In the first case, $\mathbb{Q}(\sqrt{513 / 10})$ suffers another quadratic extension, while in the second, the equation for $b$ splits over $\mathbb{Q}\left(10^{1 / 3}\right)$ in the limit $\psi \rightarrow \infty$.

What is not immediately obvious is that, upon plugging these results into the inhomogeneity (2.32), and taking into account the overall prefactor $1+2 \omega=\sqrt{-3}$, it turns out that the final result for the residue fields at $z_{\mathrm{LV}}$ is significantly simpler than at some of the intermediate steps.

Specifically, the first group of four branches has ramification index $r=2$, and residue field $K_{1}=\mathbb{Q}\left(\zeta_{1}, \zeta_{2}\right)$, where $\zeta_{1}^{2}=-2, \zeta_{2}^{2}=-57$. The A-model expansion of the normal function is

$$
\begin{aligned}
(2 \pi i)^{2} \mathcal{W}_{A} \equiv & 480120 \zeta_{1} q^{1 / 2}+2894243400 \zeta_{2} q+\frac{3072231093399320}{3} \zeta_{1} q^{3 / 2} \\
& +16749751924576485360 \zeta_{2} q^{2}+\frac{45634140857715370626589476}{5} \zeta_{1} q^{5 / 2} \\
& +192692509139523826715663010240 \zeta_{2} q^{3} \\
& +\frac{6085990469674530883728974279217064500}{49} \zeta_{1} q^{7 / 2} \\
& +2954640183071216785838740930876082745120 \zeta_{2} q^{4} \\
& +\frac{56219253156252289550103460315334373757593346895}{27} \zeta_{1} q^{9 / 2}+\cdots
\end{aligned}
$$

Note that in this case, the irrationality of the coefficients is not just an overall constant. The twist of the multi-cover formula depends on $n_{d}$

$$
\sum_{d} \tilde{n}_{d} q^{d / 2}=\zeta_{1} \sum_{k, d \text { odd }} n_{d} \frac{\left(\frac{-2}{k}\right)}{k^{2}} q^{d k / 2}+\zeta_{2} \sum_{k, d} n_{2 d} \frac{\left(\frac{-57}{k}\right)}{k^{2}} q^{d k}
$$

Moreover, as was already noticed in [1], the $n_{d}$ might not be integral at the discriminant of the extension $K_{1} / \mathbb{Q}$. Here, the denominator of $n_{d}$ for $d$ odd is a growing power of 2 . 
For the remaining six branches of our cycle, with $r=3$, the residue field in fact collapses back to $\mathbb{Q}(\sqrt{-3})$. The A-model expansion is

$$
\begin{aligned}
\frac{(2 \pi i)^{3}}{\sqrt{-3}} \mathcal{W}_{A}= & 56100 q^{1 / 3}+35413275 q^{2 / 3}+42226839000 q \\
& +\frac{264700529287425}{4} q^{4 / 3}+120847065541631256 q^{5 / 3} \\
& +243374447043299404350 q^{2}+\frac{25706778509839946246266800}{49} q^{7 / 3} \\
& +\frac{19022909901384216052391949375}{16} q^{8 / 3}+\cdots
\end{aligned}
$$

and we have an integrality structure as in (3.4).

\subsection{Quintic}

So far, all residue field extensions have had abelian Galois group. The simplest (unfortunately, not simple) example with a non-abelian Galois group that we know comes from the conics on the mirror quintic. We refer to $[1,21]$ for the full explanation of the integrality, and here content ourselves with briefly reviewing the branch structure.

As mentioned before, the situation is rather similar to that for lines on the dectic. The extension by $a$ in (2.13) splits around $\psi \rightarrow \infty$ in one of degree 2 , and one of degree 3 , which are then both extended quadratically once we add $b$. In the end, the total residue field extension for the first group is biquadratic, of the form $\mathbb{Q}(\zeta)$, with $\zeta^{4}+100 \zeta^{2}-6000=0$. The Galois group of this polynomial is the (non-abelian) dihedral group $D_{4}$. Note in particular that this extension survives in the expansion of the inhomogeneity (2.16). The A-model expansion is

$$
\begin{aligned}
(2 \pi i)^{2} \cdot \mathcal{W}_{A}= & \left(-304960000 \zeta+7227200 \zeta^{3}\right) q \\
& -\frac{512000}{51}\left(-1016270788225 \zeta+24084846092 \zeta^{3}\right) q^{2} \\
& +\frac{40000}{7803}(-131215286737935072263800 \zeta \\
& \left.+3109702672077500263451 \zeta^{3}\right) q^{3}+\cdots .
\end{aligned}
$$

The second group of conics around $z_{\mathrm{LV}}$ has $r=6$, and residue field $\mathbb{Q}\left(5^{1 / 3}\right)$

$$
\begin{aligned}
(2 \pi i)^{2} \cdot \mathcal{W}_{A}= & 2400 \cdot 5^{2 / 3} q^{1 / 6}-400600 q^{1 / 2}+\frac{120620000}{3} \cdot 5^{1 / 3} q^{5 / 6} \\
& -\frac{7863785008000}{1323} \cdot 5^{2 / 3} q^{7 / 6}+\frac{48067627724000}{9} q^{3 / 2}+\cdots
\end{aligned}
$$




\section{Integrality of monodromy}

As before, we first collect the homogeneous data, with implicit reference to the introduction of the paper.

The point of the normalization (2.4) of the holomorphic three-form is that the so-called fundamental period [5] takes a particularly compact form — the three-cycle $\gamma_{0}$, defined in a neighborhood of $z=z_{\mathrm{LV}}=0$ by encircling the coordinate axes, is invariant under $z \rightarrow e^{2 \pi i} z$ and gives the period

$$
\varpi_{0}(z)=\int_{\gamma_{0}} \Omega=\frac{1}{(2 \pi i)^{5}} \int \prod_{i} \frac{d x_{i}}{x_{i}} \sum_{n=0}^{\infty}\left(\frac{\sum \frac{w_{i}}{d} x_{i}^{d / w_{i}}}{\psi \prod x_{i}}\right)^{n}=\sum_{n=0}^{\infty} \frac{(d n) !}{\prod\left(w_{i} n\right) !} \tilde{z}^{n},
$$

where $\tilde{z}=\frac{\prod_{i} w_{i}^{w_{i}}}{d^{d}} z$. This can be readily verified to satisfy the Picard-Fuchs equation (2.6). All solutions of that equation around $z=0$ are obtained from the hypergeometric generating function

$$
\varpi(\tilde{z} ; H)=\sum_{n=0}^{\infty} \frac{\Gamma(1+d(n+H))}{\prod \Gamma\left(1+w_{i}(n+H)\right)} \tilde{z}^{n+H}
$$

by taking derivatives with respect to $H$. We define for $j=0,1,2,3$

$$
\varphi_{j}(z)=\left.\frac{1}{(2 \pi i)^{j}}\left(\partial_{H}\right)^{j}\right|_{H=0} \varpi(z ; H)
$$

In terms of these, the integral basis of periods is given by ${ }^{4}$

$$
\Pi=\left(\begin{array}{c}
\varpi_{0} \\
\varpi_{1} \\
\varpi_{2} \\
\varpi_{3}
\end{array}\right)=\left(\begin{array}{cccc}
1 & 0 & 0 & 0 \\
0 & 1 & 0 & 0 \\
-\frac{c_{2}}{24} & -\frac{\kappa}{2} & \frac{\kappa}{2} & 0 \\
\alpha & -\frac{c_{2}}{24} & 0 & -\frac{\kappa}{6}
\end{array}\right) \cdot\left(\begin{array}{c}
\varphi_{0} \\
\varphi_{1} \\
\varphi_{2} \\
\varphi_{3}
\end{array}\right) .
$$

Here, the change of basis involves some topological invariants of the mirror manifold, viz., the classical triple intersection number $\kappa=d / \prod w_{i}$, the second Chern number $c_{2}=\kappa \sum w_{i} w_{j}$ and the Euler number $\chi=\kappa\left(\sum w_{i} w_{j} w_{k}-\right.$

\footnotetext{
${ }^{4}$ This basis is almost equal to $\left.\frac{1}{j !(2 \pi i)^{j}}\left(\partial_{H}\right)^{j}\right|_{H=0} \frac{\prod \Gamma\left(1+w_{i} H\right)}{\Gamma(1+d H)} \varpi(z ; H)$, with due account of $\kappa$. Integral monodromy still prefers (4.4).
} 
$\left.d \sum w_{i} w_{j}\right)$, which enters the constant $(1.8)^{5}$

$$
\alpha=-\chi \frac{\zeta(3)}{(2 \pi i)^{3}} .
$$

With respect to this basis, the large volume and conifold monodromy are given by the matrices

$$
M_{\mathrm{LV}}=\left(\begin{array}{cccc}
1 & 0 & 0 & 0 \\
1 & 1 & 0 & 0 \\
0 & \kappa & 1 & 0 \\
-\frac{c_{2}}{12}-\frac{\kappa}{6} & -\kappa & -1 & 1
\end{array}\right), \quad M_{\mathrm{C}}=\left(\begin{array}{cccc}
1 & 0 & 0 & 1 \\
0 & 1 & 0 & 0 \\
0 & 0 & 1 & 0 \\
0 & 0 & 0 & 1
\end{array}\right)
$$

while the Gepner monodromy is

$$
M_{\mathrm{G}}=M_{\mathrm{LV}} \cdot M_{\mathrm{C}}
$$

The general strategy for calculating the extension of these matrices by the algebraic cycles is explained at the beginning of Section 4.2. First, we warm up with those somewhat simpler examples.

\subsection{Octic}

As we have seen in the previous sections, the extended moduli spaces associated with lines on the octic are rather similar to that of the van Geemen lines on the quintic, studied in [2].

4.1.1. First component. More precisely, the lines with inhomogeneity (2.29) are associated with a degree 8 covering, which is branched at $z_{\mathrm{LV}}$ with ramification index 4 and at the open string discriminant $z_{\mathrm{D}}=7^{-4}$ with index 2. In other words, at $z_{\mathrm{LV}}$, the eight branches split into two groups of four, while at $z_{\mathrm{D}}$, the eight branches come together pairwise.

To give a little more detailed account of what is going on globally, we recall that the inhomogeneity (2.29) was calculated (see appendix) over $\psi$ space as corresponding to the solution $a^{2}=3$ of (2.27). Because of the choice of root of -3 , these are really two branches of our cycle. The other branches can be reached by multiplying $a^{2}$ by a fourth root of unity, or equivalently by a monodromy $z \rightarrow e^{2 \pi i} z$.

Now notice that all interesting points in $z$-space line up conveniently on the real axis: $0=z_{\mathrm{LV}}<z_{\mathrm{D}}<z_{\mathrm{C}}<z_{\mathrm{G}}=\infty$. Therefore, it is natural to carry

\footnotetext{
${ }^{5}$ For $d=5,6,8,10, \kappa=5,3,2,1, c_{2}=50,42,44,34,-\chi=200,204,296,288$.
} 
out the monodromy calculations along the positive real axis, where we write $f_{1}$ as

$$
\frac{\sqrt{-3}}{(2 \pi i)^{2}} \cdot \frac{3}{16} \cdot \frac{z^{1 / 4}+8 z^{1 / 2}}{\left(1-7 z^{1 / 4}\right)^{5 / 2}}
$$

Let us label the lines with this calculated inhomogeneity on the positive real axis as $C_{z, 1}$ and $C_{z, 2}$ for the two choices of square-root, respectively, and the associated truncated normal functions $\tau_{1}, \tau_{2}$. Recall that it is understood implicitly that we are calculating the chain integrals with respect to some fixed globally constant cycle (e.g., a "coordinate line"). Naturally, the other lines and normal functions would be labeled $C_{z, k}, \tau_{k}$ with $k=3, \ldots, 8$, and their inhomogeneity is obtained from (4.8) by multiplying $z^{1 / 4}$ with a fourth root of unity.

In accord with this geometric situation, we can fix the ambiguity in the solution of the inhomogeneous equation, and hence recover the full $\tau_{k}$ 's, by imposing that for $k=1,2,3,4$, we have $\tau_{2 k-1}=-\tau_{2 k}$, and that they vanish at $z=z_{\mathrm{D}}$. This is the same strategy as in [2]. In the local coordinate $y=1-7 z^{1 / 4}$, we find

$$
\frac{4 \pi^{2}}{\sqrt{-3}} \tau_{1}=\frac{392}{15} y^{3 / 2}+\frac{135191}{5625} y^{5 / 2}+\frac{23856287}{1125000} y^{7 / 2}+\cdots
$$

To determine the behavior of $\tau_{1}$ at $z_{\mathrm{LV}}$, we might continue it numerically as a solution of the differential equation, or as in [2], pick a convenient point of comparison between $z_{\mathrm{LV}}$ and $z_{\mathrm{D}}$ where both the power series expansion (4.9) and

$$
\frac{(2 \pi i)^{2}}{\sqrt{-3}} \tau_{1}^{\mathrm{LV}}=48 z^{1 / 4}+\frac{153}{2} z^{1 / 2}+210 z^{3 / 4}+\frac{190365}{256} z+\cdots
$$

converge well. We find

$$
\tau_{1}=\tau_{1}^{\mathrm{LV}}+a \varpi_{0}-2 \varpi_{1}+\frac{1}{2} \varpi_{3}
$$

where $a \approx i 3.085052546678470732727 \ldots$

4.1.2. Second component. The analysis for $f_{2}$ is quite similar, the main difference being that the covering has total degree 2 and is branched only 
at $z_{\mathrm{D}}$ and $z_{\mathrm{G}}$. The expansions are

(4.12)

$$
\frac{(2 \pi i)^{2}}{\sqrt{-7}} \tau_{1}=\frac{7}{5} y^{3 / 2}+\frac{23051}{20000} y^{5 / 2}+\frac{15388807}{16000000} y^{7 / 2}+\frac{19044150391}{23040000000} y^{9 / 2}+\cdots,
$$

(where $y=1-2401 z$ ) and

$$
\frac{(2 \pi i)^{2}}{\sqrt{-7}} \tau_{1}^{\mathrm{LV}}=\frac{18963}{16} z+\frac{577705085643}{1048576} z^{2}+\frac{2125304249123593811}{4294967296} z^{3}+\cdots
$$

Analytic continuation shows

$$
\tau_{1}=\tau_{1}^{\mathrm{LV}}+a \varpi_{0}-3 \varpi_{1}+\frac{1}{2} \varpi_{3}
$$

with $a \approx i 6.48474571034689069 \ldots$

\subsection{Dectic}

We are now ready to embark on the calculation of the monodromy for the inhomogeneity (2.32). As discussed previously, the covering has total degree 10 , branched at $z_{\mathrm{LV}}, z_{\mathrm{D}_{1}}$ and $z_{\mathrm{D}_{2}}$. Again, all these branch points lie on the real axis, with

$$
0=z_{\mathrm{LV}}<z_{\mathrm{D}_{1}}\left(\approx 2.56 \cdot 10^{-5}\right)<z_{\mathrm{D}_{2}}\left(\approx 4.12 \cdot 10^{-3}\right)<z_{\mathrm{C}}=1<z_{\mathrm{G}}
$$

Recall the basic goal and strategy: we want to determine the asymptotic behavior at $z_{\mathrm{LV}}$ of the truncated normal function $\tau_{k}$ for $k=1, \ldots, 10$, associated with each branch, $C_{z, k}$ of our algebraic cycle. To this end, we need to fix the solution of the inhomogeneous Picard-Fuchs equation modulo integral periods. Pertinent information is contained in the boundary condition at the open string discriminant, and in the statement that all monodromies be integral. The simplest degeneration (which is all we have to deal with in our examples) is that two branches, say $C_{z, k_{1}}$ and $C_{z, k_{2}}$, come together at a component of the discriminant, say $z_{\mathrm{D}}$. Then the condition is that there be an integral period $p$ such that

$$
\frac{\tau_{k_{1}}-\tau_{k_{2}}-p}{\left(z-z_{\mathrm{D}}\right)^{3 / 2}}
$$

is regular at $z_{\mathrm{D}}$. As explained in [2], this condition ensures that the full normal function vanishes at $z_{\mathrm{D}}$ (in other words, not only the integral of the holomorphic three-form over a bounding three-chain, but also its derivative, 
which gives the integral of the $(2,1)$-form). Under monodromy around $z_{\mathrm{D}}$, we have

$$
\left(\tau_{k_{1}}, \tau_{k_{2}}\right) \rightarrow\left(\tau_{k_{2}}+p, \tau_{k_{1}}-p\right)
$$

We will refer to (4.16) as the "vanishing domain wall" condition. As we just saw, it ensures integrality of monodromy around the open string discriminant.

In general, integral monodromy is the statement that for each of our singular points (including open string discriminant, $z_{\mathrm{LV}}, z_{\mathrm{C}}$ and $z_{\mathrm{G}}$ ), there should be a permutation matrix $\left(\sigma_{k}^{l}\right)$ and an integral matrix $\left(A_{k}^{i}\right)$ such that upon encircling that point

$$
\tau_{k} \rightarrow \sigma_{k}^{l} \tau_{l}+A_{k}^{i} \varpi_{i}
$$

where $\left(\varpi_{i}\right)_{i=0,1,2,3}$ is the integral basis of periods (4.4). We find it convenient to combine $\left(\sigma_{k}^{l}\right)$ and $\left(A_{k}^{i}\right)$ with the matrix $M$ representing the monodromy of periods into a single matrix $\hat{M}$ of block form

$$
\hat{M}=\left(\begin{array}{cc}
\sigma & A \\
0 & M
\end{array}\right)
$$

that acts on the "extended period vector"

$$
\hat{\Pi}=\left(\tau_{1}, \ldots, \tau_{10}, \varpi_{0}, \ldots, \varpi_{3}\right)^{\mathrm{T}} .
$$

The collection of these matrices over all singular points gives the extended monodromy representation advertised in (1.16).

In the previous examples, we exploited the fact that the open string discriminant consisted of only a single point, and that there was, up to simple symmetries, essentially only one vanishing domainwall. This allowed us to fix $\tau_{k}$ at the open string discriminant, and then continue it to $z_{\mathrm{LV}}$ in order to extract the asymptotic behavior.

In the present example, we will proceed the other way around. We begin with introducing the "large volume solutions," $\tau_{k}^{\mathrm{LV}}$, distinguished by their vanishing at $z=0$. (Technically, we impose that there be no logarithmic terms, and no constant, in the solution of the differential equation.) We then calculate the monodromy of these solutions around all the singular points. This will not be integral in general, but we can improve on this by adding suitable combinations of the integral periods. Namely, there is a 
matrix $\left(B_{k}^{i}\right)$, which as it turns out is unique modulo integers, such that

$$
\tau_{k}=\tau_{k}^{\mathrm{LV}}+B_{k}^{i} \varpi_{i}
$$

has integral monodromy. At the end, we check all vanishing domainwall conditions (4.16).

Let us see what this looks like in practice. Referring to Section 3.2, we label the four branches in the first group, see Equation (3.9), such that if $\tau_{1}^{\mathrm{LV}}$ corresponds to the roots $\left(\zeta_{1}, \zeta_{2}\right)$ of $\zeta_{1}^{2}=-2, \zeta_{2}^{2}=-57$, then $\tau_{2}^{\mathrm{LV}}$ corresponds to $\left(-\zeta_{1}, \zeta_{2}\right), \tau_{3}^{\mathrm{LV}}$ to $\left(-\zeta_{1},-\zeta_{2}\right)$ and $\tau_{4}^{\mathrm{LV}}$ to $\left(\zeta_{1},-\zeta_{2}\right)$. Thus, large volume monodromy acts by exchanging $\left(\tau_{1}^{\mathrm{LV}}, \tau_{2}^{\mathrm{LV}}\right)$ and $\left(\tau_{3}^{\mathrm{LV}}, \tau_{4}^{\mathrm{LV}}\right)$.

In the second group, see Equation $(3.11), \tau_{5,6,7}^{\mathrm{LV}}$ correspond to one choice of $\sqrt{-3}$ and $\tau_{8,9,10}^{\mathrm{LV}}$ to the other, ordered in the same way such that large volume monodromy acts by cyclic permutation.

Tracking these solutions to the first component of the discriminant $z_{\mathrm{D}_{1}}$ along the positive real axis, we find that it is the combinations $\left(k_{1}, k_{2}\right)=$ $(4,7)$ and $\left(k_{1}, k_{2}\right)=(1,10)$ that should vanish there (though they do not quite yet).

Skirting around $z_{\mathrm{D}_{1}}$ in the positive upper half plane, we proceed to $z_{\mathrm{D}_{2}}$ and find that the vanishing domainwall there will come from the combination $\left(k_{1}, k_{2}\right)=(2,3)$.

Finally, we head for the conifold, encircle it in the positive direction and return to $z_{\mathrm{LV}}$ along the same path. The net result is that the $\tau_{k}^{\mathrm{LV}}$ pick up a complex multiple of the fundamental period $\varpi_{0}$, in the above order given by

$$
\begin{aligned}
& \left(\frac{9}{2}+a_{1},-3+a_{1}, 3-a_{1},-\frac{9}{2}-a_{1},-\frac{11}{6}+a_{2}, \frac{11}{6}+a_{2}, \frac{9}{2}+a_{2}, \frac{11}{6}\right. \\
& \left.\quad-a_{2},-\frac{11}{6}-a_{2},-\frac{9}{2}-a_{2}\right),
\end{aligned}
$$

where $a_{1} \approx i 5.154774632407 \ldots$ and $a_{2} \approx i 5.090336702019 \ldots$..

It is then not hard to check that the change of basis (4.21), with $B$ given by

$$
B^{\mathrm{T}}=\left(\begin{array}{cccccccccc}
-\frac{1}{2}-a_{1} & -a_{1} & a_{1}-\frac{1}{2}+a_{1} & -\frac{1}{6}-a_{2} & \frac{1}{6}-a_{2} & \frac{1}{2}-a_{2} & \frac{1}{6}+a_{2} & -\frac{1}{6}+a_{2}-\frac{1}{2}+a_{2} \\
\frac{1}{2} & \frac{1}{2} & \frac{1}{2} & \frac{1}{2} & \frac{2}{3} & \frac{2}{3} & \frac{2}{3} & \frac{1}{3} & \frac{1}{3} & \frac{1}{3} \\
0 & 0 & 0 & 0 & 0 & 0 & 0 & 0 & 0 & 0 \\
0 & 0 & 0 & 0 & 0 & 0 & 0 & 0 & 0 & 0
\end{array}\right)
$$

makes the conifold monodromy integral in a way that is consistent with the extension at large volume. Specifically, in this basis the extended monodromy 
matrices are

$$
\hat{M}_{\mathrm{LV}}=\left(\begin{array}{cccccccccc|cccc}
0 & 1 & 0 & 0 & 0 & 0 & 0 & 0 & 0 & 0 & 0 & 0 & 0 & 0 \\
1 & 0 & 0 & 0 & 0 & 0 & 0 & 0 & 0 & 0 & 1 & 0 & 0 & 0 \\
0 & 0 & 0 & 1 & 0 & 0 & 0 & 0 & 0 & 0 & 1 & 0 & 0 & 0 \\
0 & 0 & 1 & 0 & 0 & 0 & 0 & 0 & 0 & 0 & 0 & 0 & 0 & 0 \\
0 & 0 & 0 & 0 & 0 & 0 & 1 & 0 & 0 & 0 & 0 & 0 & 0 & 0 \\
0 & 0 & 0 & 0 & 1 & 0 & 0 & 0 & 0 & 0 & 1 & 0 & 0 & 0 \\
0 & 0 & 0 & 0 & 0 & 1 & 0 & 0 & 0 & 0 & 1 & 0 & 0 & 0 \\
0 & 0 & 0 & 0 & 0 & 0 & 0 & 0 & 0 & 1 & 1 & 0 & 0 & 0 \\
0 & 0 & 0 & 0 & 0 & 0 & 0 & 1 & 0 & 0 & 0 & 0 & 0 & 0 \\
0 & 0 & 0 & 0 & 0 & 0 & 0 & 0 & 1 & 0 & 0 & 0 & 0 & 0 \\
\hline 0 & 0 & 0 & 0 & 0 & 0 & 0 & 0 & 0 & 0 & 1 & 0 & 0 & 0 \\
0 & 0 & 0 & 0 & 0 & 0 & 0 & 0 & 0 & 0 & 1 & 1 & 0 & 0 \\
0 & 0 & 0 & 0 & 0 & 0 & 0 & 0 & 0 & 0 & 0 & 1 & 1 & 0 \\
0 & 0 & 0 & 0 & 0 & 0 & 0 & 0 & 0 & 0 & -3 & -1 & -1 & 1
\end{array}\right), \quad \hat{M}_{\mathrm{C}}=\left(\begin{array}{cccccccccc|cccc}
1 & 0 & 0 & 0 & 0 & 0 & 0 & 0 & 0 & 0 & 0 & 0 & 0 & 4 \\
0 & 1 & 0 & 0 & 0 & 0 & 0 & 0 & 0 & 0 & 0 & 0 & 0 & -3 \\
0 & 0 & 1 & 0 & 0 & 0 & 0 & 0 & 0 & 0 & 0 & 0 & 0 & 3 \\
0 & 0 & 0 & 1 & 0 & 0 & 0 & 0 & 0 & 0 & 0 & 0 & 0 & -5 \\
0 & 0 & 0 & 0 & 1 & 0 & 0 & 0 & 0 & 0 & 0 & 0 & 0 & -2 \\
0 & 0 & 0 & 0 & 0 & 1 & 0 & 0 & 0 & 0 & 0 & 0 & 0 & 2 \\
0 & 0 & 0 & 0 & 0 & 0 & 1 & 0 & 0 & 0 & 0 & 0 & 0 & 5 \\
0 & 0 & 0 & 0 & 0 & 0 & 0 & 1 & 0 & 0 & 0 & 0 & 0 & 2 \\
0 & 0 & 0 & 0 & 0 & 0 & 0 & 0 & 1 & 0 & 0 & 0 & 0 & -2 \\
0 & 0 & 0 & 0 & 0 & 0 & 0 & 0 & 0 & 1 & 0 & 0 & 0 & -5 \\
\hline 0 & 0 & 0 & 0 & 0 & 0 & 0 & 0 & 0 & 0 & 1 & 0 & 0 & 1 \\
0 & 0 & 0 & 0 & 0 & 0 & 0 & 0 & 0 & 0 & 0 & 1 & 0 & 0 \\
0 & 0 & 0 & 0 & 0 & 0 & 0 & 0 & 0 & 0 & 0 & 0 & 1 & 0 \\
0 & 0 & 0 & 0 & 0 & 0 & 0 & 0 & 0 & 0 & 0 & 0 & 0 & 1
\end{array}\right),
$$

$$
\hat{M}_{\mathrm{D}_{1}}=\left(\begin{array}{cccccccccc|cccc}
0 & 0 & 0 & 0 & 0 & 0 & 0 & 0 & 0 & 1 & 0 & -4 & 0 & 1 \\
0 & 1 & 0 & 0 & 0 & 0 & 0 & 0 & 0 & 0 & 0 & 0 & 0 & 0 \\
0 & 0 & 1 & 0 & 0 & 0 & 0 & 0 & 0 & 0 & 0 & 0 & 0 & 0 \\
0 & 0 & 0 & 0 & 0 & 0 & 1 & 0 & 0 & 0 & -1 & 4 & 0 & -1 \\
0 & 0 & 0 & 0 & 1 & 0 & 0 & 0 & 0 & 0 & 0 & 0 & 0 & 0 \\
0 & 0 & 0 & 0 & 0 & 1 & 0 & 0 & 0 & 0 & 0 & 0 & 0 & 0 \\
0 & 0 & 0 & 1 & 0 & 0 & 0 & 0 & 0 & 0 & 1 & -4 & 0 & 1 \\
0 & 0 & 0 & 0 & 0 & 0 & 0 & 1 & 0 & 0 & 0 & 0 & 0 & 0 \\
0 & 0 & 0 & 0 & 0 & 0 & 0 & 0 & 1 & 0 & 0 & 0 & 0 & 0 \\
1 & 0 & 0 & 0 & 0 & 0 & 0 & 0 & 0 & 0 & 0 & 4 & 0 & -1 \\
\hline 0 & 0 & 0 & 0 & 0 & 0 & 0 & 0 & 0 & 0 & 1 & 0 & 0 & 0 \\
0 & 0 & 0 & 0 & 0 & 0 & 0 & 0 & 0 & 0 & 0 & 1 & 0 & 0 \\
0 & 0 & 0 & 0 & 0 & 0 & 0 & 0 & 0 & 0 & 0 & 0 & 1 & 0 \\
0 & 0 & 0 & 0 & 0 & 0 & 0 & 0 & 0 & 0 & 0 & 0 & 0 & 1
\end{array}\right), \quad \hat{M}_{\mathrm{D}_{2}}=\left(\begin{array}{cccccccccc|cccc}
1 & 0 & 0 & 0 & 0 & 0 & 0 & 0 & 0 & 0 & 0 & 0 & 0 & 0 \\
0 & 0 & 1 & 0 & 0 & 0 & 0 & 0 & 0 & 0 & 0 & -3 & 0 & 0 \\
0 & 1 & 0 & 0 & 0 & 0 & 0 & 0 & 0 & 0 & 0 & 3 & 0 & 0 \\
0 & 0 & 0 & 1 & 0 & 0 & 0 & 0 & 0 & 0 & 0 & 0 & 0 & 0 \\
0 & 0 & 0 & 0 & 1 & 0 & 0 & 0 & 0 & 0 & 0 & 0 & 0 & 0 \\
0 & 0 & 0 & 0 & 0 & 1 & 0 & 0 & 0 & 0 & 0 & 0 & 0 & 0 \\
0 & 0 & 0 & 0 & 0 & 0 & 1 & 0 & 0 & 0 & 0 & 0 & 0 & 0 \\
0 & 0 & 0 & 0 & 0 & 0 & 0 & 1 & 0 & 0 & 0 & 0 & 0 & 0 \\
0 & 0 & 0 & 0 & 0 & 0 & 0 & 0 & 1 & 0 & 0 & 0 & 0 & 0 \\
0 & 0 & 0 & 0 & 0 & 0 & 0 & 0 & 0 & 1 & 0 & 0 & 0 & 0 \\
\hline 0 & 0 & 0 & 0 & 0 & 0 & 0 & 0 & 0 & 0 & 1 & 0 & 0 & 0 \\
0 & 0 & 0 & 0 & 0 & 0 & 0 & 0 & 0 & 0 & 0 & 1 & 0 & 0 \\
0 & 0 & 0 & 0 & 0 & 0 & 0 & 0 & 0 & 0 & 0 & 0 & 1 & 0 \\
0 & 0 & 0 & 0 & 0 & 0 & 0 & 0 & 0 & 0 & 0 & 0 & 0 & 1
\end{array}\right) .
$$

One may then first of all make the consistency check that the extension of (4.7)

$$
\hat{M}_{\mathrm{G}}=\hat{M}_{\mathrm{LV}} \cdot \hat{M}_{\mathrm{D}_{1}} \cdot \hat{M}_{\mathrm{D}_{2}} \cdot \hat{M}_{\mathrm{C}}
$$

(remember the lineup (4.15) and that these matrices compose on the right) satisfies

$$
\left(\hat{M}_{\mathrm{G}}\right)^{10}=1 \text {. }
$$

Finally, we verify the existence of vanishing domain walls. We find that

$$
\tau_{7}-\tau_{4}-\varpi_{0}+4 \varpi_{1}-\varpi_{3} \quad \text { and } \quad \tau_{10}-\tau_{1}-4 \varpi_{1}+\varpi_{3}
$$

vanish at $z_{\mathrm{D}_{1}}$, and

$$
\tau_{2}-\tau_{3}+3 \varpi_{1}
$$

vanishes at $z_{\mathrm{D}_{2}}$. So everything appears in order.

\subsection{Quintic}

We now repeat those calculations for the extension (2.16) of the quintic. The first thing to note is that while the components of the open string 
discriminant (2.17) are still all on the real axis, one of them is negative. Namely,

$$
z_{\mathrm{D}_{11}}\left(\approx-1.76 \cdot 10^{-5}\right)<z_{\mathrm{LV}}<z_{\mathrm{D}_{2}}\left(\approx 2.34 \cdot 10^{-2}\right)<z_{\mathrm{C}}<z_{\mathrm{D}_{12}}(\approx 4.92) .
$$

We label the four branches in the first group Equation (3.12) such that $\tau_{1}, \tau_{4}$ correspond to the real roots of $\zeta^{4}+100 \zeta^{2}-6000$ and $\tau_{2}, \tau_{3}$ to the imaginary roots. Large volume monodromy leaves these branches untouched. Those in the second group, (3.13), are arranged by Mathematica such that large volume monodromy acts by $(5,6,7,8,9,10) \rightarrow(7,5,9,6,10,8)$. Under conifold monodromy, these solutions pick up the fundamental period times

$$
\left(a_{1}, 24-a_{2},-24+a_{2},-a_{1},-\frac{59}{2},-\frac{69}{2},-\frac{69}{2}, \frac{69}{2}, \frac{69}{2}, \frac{59}{2}\right)
$$

with $a_{1} \approx 1.6377482972 \ldots$ and $a_{2} \approx i 93.620780658 \ldots$. To compensate for this, we add the periods

$$
B^{\mathrm{T}}=\left(\begin{array}{cccccccccc}
-a_{1} & -24+a_{2} & 24-a_{2} & a_{1} & \frac{59}{2} & \frac{69}{2} & \frac{69}{2} & -\frac{69}{2} & -\frac{69}{2} & -\frac{59}{2} \\
0 & 0 & 0 & 0 & 0 & 0 & 0 & 0 & 0 & 0 \\
0 & 0 & 0 & 0 & 0 & 0 & 0 & 0 & 0 & 0 \\
0 & 0 & 0 & 0 & 0 & 0 & 0 & 0 & 0 & 0
\end{array}\right)
$$

giving the monodromy matrices

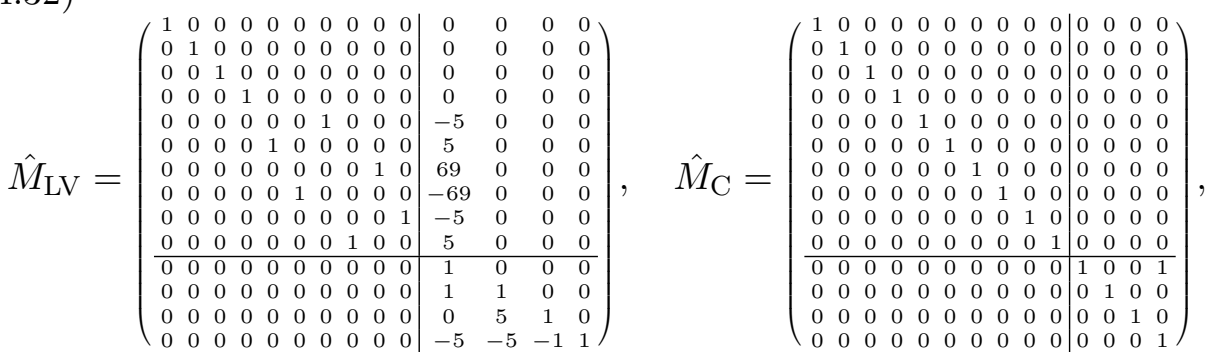

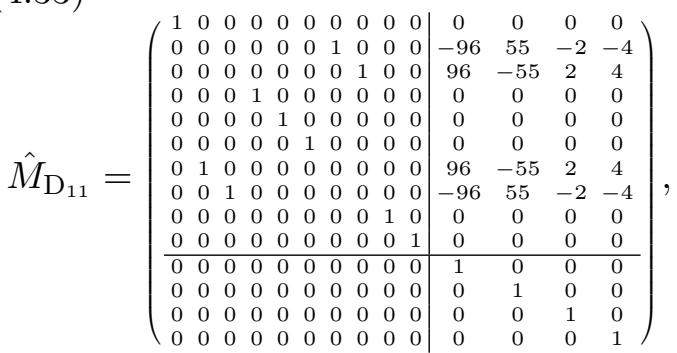




$$
\hat{M}_{\mathrm{D}_{12}}=\left(\begin{array}{cccccccccc|cccc}
0 & 0 & 1 & 0 & 0 & 0 & 0 & 0 & 0 & 0 & -16 & 40 & 0 & -8 \\
0 & 0 & 0 & 1 & 0 & 0 & 0 & 0 & 0 & 0 & -16 & 40 & 0 & -8 \\
1 & 0 & 0 & 0 & 0 & 0 & 0 & 0 & 0 & 0 & 16 & -40 & 0 & 8 \\
0 & 1 & 0 & 0 & 0 & 0 & 0 & 0 & 0 & 0 & 16 & -40 & 0 & 8 \\
0 & 0 & 0 & 0 & 1 & 0 & 0 & 0 & 0 & 0 & 0 & 0 & 0 & 0 \\
0 & 0 & 0 & 0 & 0 & 1 & 0 & 0 & 0 & 0 & 0 & 0 & 0 & 0 \\
0 & 0 & 0 & 0 & 0 & 0 & 1 & 0 & 0 & 0 & 0 & 0 & 0 & 0 \\
0 & 0 & 0 & 0 & 0 & 0 & 0 & 1 & 0 & 0 & 0 & 0 & 0 & 0 \\
0 & 0 & 0 & 0 & 0 & 0 & 0 & 0 & 1 & 0 & 0 & 0 & 0 & 0 \\
0 & 0 & 0 & 0 & 0 & 0 & 0 & 0 & 0 & 1 & 0 & 0 & 0 & 0 \\
\hline 0 & 0 & 0 & 0 & 0 & 0 & 0 & 0 & 0 & 0 & 1 & 0 & 0 & 0 \\
0 & 0 & 0 & 0 & 0 & 0 & 0 & 0 & 0 & 0 & 0 & 1 & 0 & 0 \\
0 & 0 & 0 & 0 & 0 & 0 & 0 & 0 & 0 & 0 & 0 & 0 & 1 & 0 \\
0 & 0 & 0 & 0 & 0 & 0 & 0 & 0 & 0 & 0 & 0 & 0 & 0 & 1
\end{array}\right),
$$

$$
\hat{M}_{\mathrm{D}_{2}}=\left(\begin{array}{cccccccccc|cccc}
1 & 0 & 0 & 0 & 0 & 0 & 0 & 0 & 0 & 0 & 0 & 0 & 0 & 0 \\
0 & 0 & 1 & 0 & 0 & 0 & 0 & 0 & 0 & 0 & -48 & 80 & 0 & 0 \\
0 & 1 & 0 & 0 & 0 & 0 & 0 & 0 & 0 & 0 & 48 & -80 & 0 & 0 \\
0 & 0 & 0 & 1 & 0 & 0 & 0 & 0 & 0 & 0 & 0 & 0 & 0 & 0 \\
0 & 0 & 0 & 0 & 1 & 0 & 0 & 0 & 0 & 0 & 0 & 0 & 0 & 0 \\
0 & 0 & 0 & 0 & 0 & 1 & 0 & 0 & 0 & 0 & 0 & 0 & 0 & 0 \\
0 & 0 & 0 & 0 & 0 & 0 & 1 & 0 & 0 & 0 & 0 & 0 & 0 & 0 \\
0 & 0 & 0 & 0 & 0 & 0 & 0 & 1 & 0 & 0 & 0 & 0 & 0 & 0 \\
0 & 0 & 0 & 0 & 0 & 0 & 0 & 0 & 1 & 0 & 0 & 0 & 0 & 0 \\
0 & 0 & 0 & 0 & 0 & 0 & 0 & 0 & 0 & 1 & 0 & 0 & 0 & 0 \\
\hline 0 & 0 & 0 & 0 & 0 & 0 & 0 & 0 & 0 & 0 & 1 & 0 & 0 & 0 \\
0 & 0 & 0 & 0 & 0 & 0 & 0 & 0 & 0 & 0 & 0 & 1 & 0 & 0 \\
0 & 0 & 0 & 0 & 0 & 0 & 0 & 0 & 0 & 0 & 0 & 0 & 1 & 0 \\
0 & 0 & 0 & 0 & 0 & 0 & 0 & 0 & 0 & 0 & 0 & 0 & 0 & 1
\end{array}\right) .
$$

By the ordering (4.29), the extended Gepner monodromy is given by

$$
\hat{M}_{\mathrm{G}}=\hat{M}_{\mathrm{D}_{11}} \cdot \hat{M}_{\mathrm{LV}} \cdot \hat{M}_{\mathrm{D}_{2}} \cdot \hat{M}_{\mathrm{C}} \cdot \hat{M}_{\mathrm{D}_{12}}, \quad\left(\hat{M}_{\mathrm{G}}\right)^{5}=1
$$

We also find the vanishing domain walls

$$
\begin{aligned}
\tau_{7} & -\tau_{2}-96 \varpi_{0}+55 \varpi_{1}-2 \varpi_{2}-4 \varpi_{3} \quad \text { and } \quad \tau_{8}-\tau_{3}+96 \varpi_{0} \\
& -55 \varpi_{1}+2 \varpi_{2}+4 \varpi_{3}
\end{aligned}
$$

at $z_{\mathrm{D}_{11}}$,

$$
\tau_{1}-\tau_{3}+16 \varpi_{0}-40 \varpi_{1}+8 \varpi_{3} \quad \text { and } \quad \tau_{4}-\tau_{2}-16 \varpi_{0}+40 \varpi_{1}-8 \varpi_{3}
$$

at $z_{\mathrm{D}_{12}}$ and

$$
\tau_{3}-\tau_{2}-48 \varpi_{0}+80 \varpi_{1}
$$

at $z_{\mathrm{D}_{2}}$.

\section{Discussion}

In this work, we have studied analytic invariants of a variety of (mostly new!) algebraic cycles on four one-parameter Calabi-Yau hypersurfaces in 
weighted projective space. We found these cycles by looking for holomorphic curves invariant under particular permutation symmetries of the homogeneous coordinates. In Section 2, we calculated the inhomogeneous PicardFuchs equation satisfied by the truncated normal function associated with each cycle. In Section 3, we verified that the large volume expansion satisfies the algebraic "D-logarithm" integrality of $[1,21]$. In Section 3, we calculated the monodromy representation (1.16) underlying the variation of mixed Hodge structure. A by-product of these calculations is the limiting value of the normal function. We summarize our results in table 1. (For the van Geemen lines on the quintic, we have rescaled the results of [2] by a factor of 4 in order to conform to our present conventions.)

The key formula to discuss the arithmetic data is the large volume expansion $(3.2)$

$$
\mathcal{W}_{A}=\frac{s}{2 \pi i r} \log q+a+\frac{1}{(2 \pi i)^{2}} \sum_{d=1}^{\infty} \tilde{n}_{d} q^{d / r}
$$

Namely, $r$ is the ramification (or "Puiseux") index of the corresponding branch of the covering (1.9). The coefficients $\tilde{n}_{d}$ are algebraic numbers in a finite extension of $\mathbb{Q}$, the "residue field," $K$. In our terminology, we have referred to branches with the same $r$ and $K$ as "a group of branches." The various branches in one group are distinguished by the choice of phase of $q^{1 / r}$, as well as the choice of an embedding of $K$ into $\mathbb{C}$. (We might emphasize that there are cases in which the two choices are not independent. For instance, the "second group" of conics on the quintic (3.13) has $K=\mathbb{Q}\left(5^{1 / 3}\right)$, and $r=6$. But as the coefficients satisfy $\tilde{n}_{d} / 5^{2 d / 3} \in \mathbb{Q}$, only the overall choice of phase of $5^{2 / 3} q^{1 / 6}$ matters, and there are really six branches in that group. In contrast, the first component of lines on the octic (3.3), has $r=4$ and $K=$ $\mathbb{Q}(\sqrt{-3})$. But the two choices are independent, and there are eight different branches. Somewhat more formally, we can have an embedding of the Galois group of the local extension of moduli $(\cong \mathbb{Z} / r \mathbb{Z})$ into the Galois group of the extension of residue field (or rather its Galois closure). Another example of this is the first group on the dectic, Equation (3.9).) The generator of this embedding is written as $\zeta$ in the table. We emphasize the two most important aspects of the expansion (5.1).

1) The coefficients $\tilde{n}_{d}$ satisfy "Ooguri-Vafa integrality with an arithmetic twist." In the simplest cases, this means that there is a Dirichlet 


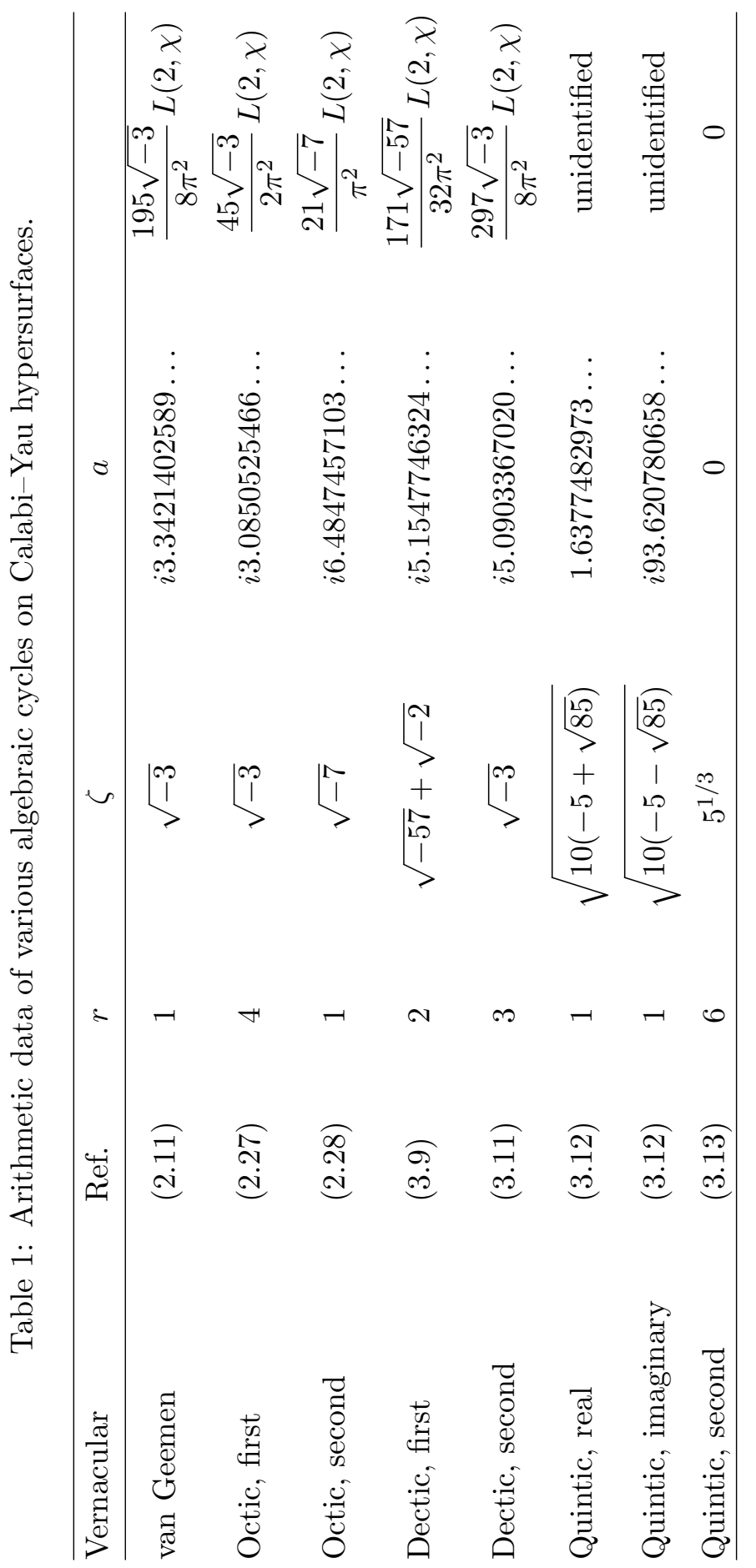


character $\chi$ such that in

$$
\sum \tilde{n}_{d} q^{d / r}=\sum n_{d} \operatorname{Li}_{2}^{(\chi)}\left(q^{d / r}\right)
$$

the $n_{d}$ are integral (at least outside the discriminant), where

$$
\operatorname{Li}_{2}^{(\chi)}(q)=\sum \frac{\chi(k)}{k^{2}} q^{k}
$$

is the "D-logarithm." Specifically, for a quadratic extension $\zeta=\sqrt{\Delta}$, we can write $\chi$ in terms of the Jacobi-symbol

$$
\chi(k)=\left(\frac{\Delta}{k}\right) .
$$

In more complicated cases, the twist depends on $n_{d}$, see around Equation (3.10) and $[1,21]$.

2) The constant term $a$ of the expansion is an interesting (conjecturally transcendental) number that we identify with the limiting value of the Abel-Jacobi map discussed in [16]. (Comparison with Section 4 will show that in many cases we have stripped off a simple rational additive that appears to be explained by the branch structure at $z_{\mathrm{LV}}$, rather than the intrinsic arithmetic of the residue field.) As we have written in the table, in all cases with abelian Galois group, $a$ can be expressed in terms of the Dirichlet L-function with the same character (5.4) that appears in the D-logarithm. We have not yet succeeded in identifying an analogous formula in the non-abelian case (first group of conics on the quintic). (The fact that $a$ vanishes (modulo $\mathbb{Q}$ ) for the second group of conics on the quintic, and that the extension by $\sqrt{-2}$ disappears from $a$ in the first group on the dectic are consequences of the interplay with the extension of moduli at $z_{\mathrm{LV}}$ that we mentioned above.)

An interesting technical aspect of our calculations is that for each globally well-defined cycle $\mathcal{C} \rightarrow B$ (cf. (1.9)), the number of independent limiting values that have to be calculated matches the number of components of the open string discriminant. For instance, for the lines on the dectic, there are two groups at $z_{\mathrm{LV}}$, and $D$ has two components. The conics on the quintic also split into two groups at $z_{\mathrm{LV}}$. However, because the residue field of the first group has two essentially independent embeddings into $\mathbb{C}$ (corresponding to the real and imaginary $\zeta$ ), we really have three independent values to 
calculate (counting one for $\mathbb{Q}\left(5^{1 / 3}\right)$ ). This is matched precisely by the fact that the open string discriminant has three different components, see (2.17). This state of affairs has allowed us to calculate $a$ in two independent ways, from the conifold monodromy and the vanishing domain wall condition. We suspect that there is an underlying general statement.

Considering which number fields appear in the examples, one might observe that lines only come with abelian extensions of $\mathbb{Q}$, though it is hard for us to tell whether this had to be true. The hunch that conics give at most solvable Galois groups is dispelled by an example from [1] (see table 1 there).

Clearly the most interesting open problem is to find an A-model explanation for the interesting arithmetic that we have observed here in the B-model. Some possibilities for the constant $a$ were mentioned in [2], and one can be rather hopeful that one of them will materialize soon. For the D-logarithm integrality, we refer to the speculations in [1].

\section{Acknowledgments}

J.W. thanks the organizers of the Workshop "Recent advances in Hodge theory," University of British Columbia, June 2013, for the invitation to present some of these results. This research is supported in part by an NSERC discovery grant and a Tier II Canada Research Chair.

\section{Appendix. Some details of residue calculation}

For the convenience of the reader, we give a few details of the residue algorithm developed in $[13,15]$, applied to the first component of $\mathbb{Z}_{3}$-invariant lines on the octic, see Equation (2.27).

We start from the expression Equation (2.4) for the holomorphic threeform. With $z=\psi^{-8}$, the Picard-Fuchs operator (2.6) has the form

$$
\begin{aligned}
\mathcal{L}= & \theta^{4}-z\left(\theta+\frac{1}{8}\right)\left(\theta+\frac{3}{8}\right)\left(\theta+\frac{5}{8}\right)\left(\theta+\frac{7}{8}\right) \\
= & \frac{1}{8^{4} \psi^{3}}\left(\left(\psi^{8}-1\right) \partial_{\psi}^{4}+\left(10 \psi^{7}+\frac{6}{\psi}\right) \partial_{\psi}^{3}+\left(25 \psi^{6}-\frac{15}{\psi^{2}}\right) \partial_{\psi}^{2}\right. \\
& \left.+\left(15 \psi^{5}+\frac{15}{\psi^{3}}\right) \partial_{\psi}+\psi^{4}\right) \frac{1}{\psi} .
\end{aligned}
$$

The Griffiths-Dwork reduction method now allows us to write the two-form in $(2.5)$ as

$$
\beta=\operatorname{Res} \tilde{\beta}
$$


where $\tilde{\beta}$ is the meromorphic three-form ${ }^{6}$

(A.3)

$$
\begin{aligned}
& \tilde{\beta}=-\frac{6 \omega_{5} x_{1}^{4} x_{2}^{4} x_{3}^{4} x_{4}^{4} x_{5}^{3}}{W^{4}}-\frac{6 \psi \omega_{5} x_{1}^{5} x_{2}^{5} x_{3}^{5} x_{4}^{5} x_{5}^{2}}{W^{4}}-\frac{6 \psi^{2} \omega_{5} x_{1}^{6} x_{2}^{6} x_{3}^{6} x_{4}^{6} x_{5}}{W^{4}}-\frac{6 \psi^{3} \omega_{1} x_{2}^{7} x_{3}^{7} x_{4}^{7} x_{5}}{W^{4}} \\
& -\frac{6 \psi^{4} \omega_{2} x_{2} x_{3}^{8} x_{4}^{8} x_{5}^{2}}{W^{4}}-\frac{6 \psi^{5} \omega_{3} x_{1} x_{2} x_{3}^{2} x_{4}^{9} x_{5}^{3}}{W^{4}}-\frac{6 \psi^{6} \omega_{4} x_{1}^{2} x_{2}^{2} x_{3}^{2} x_{4}^{3} x_{5}^{4}}{W^{4}}-\frac{6 \psi^{7} \omega_{5} x_{1}^{3} x_{2}^{3} x_{3}^{3} x_{4}^{3} x_{5}^{4}}{W^{4}} \\
& +\frac{6 \omega_{5} x_{1}^{3} x_{2}^{3} x_{3}^{3} x_{4}^{3} x_{5}^{2}}{\psi W^{3}}+\frac{4 \omega_{5} x_{1}^{4} x_{2}^{4} x_{3}^{4} x_{4}^{4} x_{5}}{W^{3}}+\frac{4 \omega_{5} x_{1}^{3} x_{2}^{3} x_{3}^{3} x_{4}^{3} x_{5}^{2}}{\psi W^{3}}+\frac{2 \psi \omega_{5} x_{1}^{5} x_{2}^{5} x_{3}^{5} x_{4}^{5}}{W^{3}} \\
& +\frac{2 \omega_{5} x_{1}^{4} x_{2}^{4} x_{3}^{4} x_{4}^{4} x_{5}}{W^{3}}+\frac{2 \omega_{5} x_{1}^{3} x_{2}^{3} x_{3}^{3} x_{4}^{3} x_{5}^{2}}{\psi W^{3}}-\frac{2 \psi^{4} \omega_{3} x_{3} x_{4}^{8} x_{5}^{2}}{W^{3}}-\frac{2 \psi^{5} \omega_{4} x_{1} x_{2} x_{3} x_{4}^{2} x_{5}^{3}}{W^{3}} \\
& -\frac{2 \psi^{6} \omega_{5} x_{1}^{2} x_{2}^{2} x_{3}^{2} x_{4}^{2} x_{5}^{3}}{W^{3}}-\frac{4 \psi^{5} \omega_{4} x_{1} x_{2} x_{3} x_{4}^{2} x_{5}^{3}}{W^{3}}-\frac{4 \psi^{6} \omega_{5} x_{1}^{2} x_{2}^{2} x_{3}^{2} x_{4}^{2} x_{5}^{3}}{W^{3}}-\frac{6 \psi^{6} \omega_{5} x_{1}^{2} x_{2}^{2} x_{3}^{2} x_{4}^{2} x_{5}^{3}}{W^{3}} \\
& -\frac{6 \omega_{5} x_{1}^{2} x_{2}^{2} x_{3}^{2} x_{4}^{2} x_{5}}{\psi^{2} W^{2}}-\frac{2 \omega_{5} x_{1}^{3} x_{2}^{3} x_{3}^{3} x_{4}^{3}}{\psi W^{2}}-\frac{2 \omega_{5} x_{1}^{2} x_{2}^{2} x_{3}^{2} x_{4}^{2} x_{5}}{\psi^{2} W^{2}}-\frac{4 \omega_{5} x_{1}^{2} x_{2}^{2} x_{3}^{2} x_{4}^{2} x_{5}}{\psi^{2} W^{2}}-\frac{\omega_{5} x_{1}^{3} x_{2}^{3} x_{3}^{3} x_{4}^{3}}{\psi W^{2}} \\
& -\frac{\omega_{5} x_{1}^{2} x_{2}^{2} x_{3}^{2} x_{4}^{2} x_{5}}{\psi^{2} W^{2}}-\frac{2 \omega_{5} x_{1}^{2} x_{2}^{2} x_{3}^{2} x_{4}^{2} x_{5}}{\psi^{2} W^{2}}-\frac{\psi^{4} \omega_{4} x_{4} x_{5}^{2}}{W^{2}}-\frac{\psi^{5} \omega_{5} x_{1} x_{2} x_{3} x_{4} x_{5}^{2}}{W^{2}} \\
& -\frac{2 \psi^{5} \omega_{5} x_{1} x_{2} x_{3} x_{4} x_{5}^{2}}{W^{2}}-\frac{4 \psi^{5} \omega_{5} x_{1} x_{2} x_{3} x_{4} x_{5}^{2}}{W^{2}}+\frac{6 \omega_{5} x_{1} x_{2} x_{3} x_{4}}{\psi^{3} W}+\frac{2 \omega_{5} x_{1} x_{2} x_{3} x_{4}}{\psi^{3} W} \\
& +\frac{4 \omega_{5} x_{1} x_{2} x_{3} x_{4}}{\psi^{3} W}+\frac{\omega_{5} x_{1} x_{2} x_{3} x_{4}}{\psi^{3} W}+\frac{2 \omega_{5} x_{1} x_{2} x_{3} x_{4}}{\psi^{3} W}-\frac{\psi^{4} \omega_{5} x_{5}}{W}
\end{aligned}
$$

and for $i=1, \ldots, 5$

$$
\omega_{i}=\omega\left(\partial_{i}\right)
$$

Now consider the line $C$ parameterized as in (2.26), with $a^{2}=3, c=b$, and $b^{2}-2 a b \psi+21=0$. To calculate the inhomogeneous Picard-Fuchs equation associated to $C$, we choose a three-chain $\Gamma$ with $\partial \Gamma=C$, and then apply Griffiths' "tube-over-cycle map" to write

$$
\mathcal{L} \int^{C} \Omega=\mathcal{L} \int_{T_{\epsilon}(\Gamma)} \tilde{\Omega} .
$$

The calculation in $\mathbb{P}^{4}$ then splits in two types of contributions: "the exact terms,"

$$
f_{\text {exact }}=\int_{T_{\epsilon}(C)} \tilde{\beta}
$$

and the "direct terms," $f_{\text {direct }}$, which come from differentiating the tube over the three-chain. If $n_{\psi}$ is the normal vector to $T_{\epsilon}(C)$ implementing infinitesimal variation in $\psi$ direction, $f_{\text {direct }}$ is obtained from (A.1) by replacing $\partial_{\psi}^{k}$

\footnotetext{
${ }^{6}$ Multiplied with $8^{4} \psi^{3}$ and up to factors of $2 \pi i$.
} 
with

$$
\partial_{\psi}^{k} \int_{T_{\epsilon}(\Gamma)} \tilde{\Omega}-\int_{T_{\epsilon}(\Gamma)} \partial_{\psi}^{k} \tilde{\Omega}=\sum_{j=0}^{k-1} \partial_{\psi}^{k-1-j} \int_{T_{\epsilon}(C)}\left(\partial_{\psi}^{j} \tilde{\Omega}\right)\left(n_{\psi}\right)
$$

We emphasize that while the final result is well-defined and does not depend on any choices (such as, the three-form $\tilde{\beta}$, or the tube $T_{\epsilon}(C)$ ), the decomposition into $f_{\text {exact }}$ and $f_{\text {direct }}$ in general will.

The freedom in laying the tube is the key to calculating the integrals defining $f_{\text {exact }}$ and $f_{\text {direct }}$ in terms of residues. Fix some choice of plane $P$ passing through $C$, and denote the residual curve by $R$

$$
\{W=0\} \cap P=C \cup R .
$$

We can now lay the tube over $C$ inside of $P$ except for some neighborhood of the intersection points

$$
C \cap R=\left\{p_{1}, \ldots, p_{s}\right\}
$$

where we have to escape into the rest of $\mathbb{P}^{4}$. A certain advantage of the lines over the conics studied in $[1,13]$ is that we have some choice in picking $P$, whereas a conic already spans a plane. In the case at hand, it turns out convenient to let the second generator point in the $x_{5}$ direction, which results in the four intersection points

$$
p_{1}=\{u=0\}, \quad p_{2}=\{u+v=0\}, \quad p_{3,4}=\left\{u^{2}-u v+v^{2}=0\right\} .
$$

Then, for each of those four points, we choose a third direction, normal to the plane, and a local coordinate $z$ on the curve. We also need a real function $f(r)$ that smoothly decreases from 1 to 0 as $r=|z|$ runs from 0 to some small positive $r_{*}$. The role of $f(r)$ is to return the tube to $P$ for $|z|>r_{*}$.

To be completely explicit, around $p_{1}$ above, with $z=u / v$, we escape into the $x_{1}$-direction. The tube is parameterized as

$$
\begin{aligned}
& x_{1}=1+z+\epsilon r g(r), \quad x_{2}=\omega+z, \quad x_{3}=\omega^{2}+z, \quad x_{4}=a z, \\
& x_{5}=c z+b z^{4}+\epsilon\left[\frac{r}{(b-a \psi) z}-\frac{7 r g(r)}{b-a \psi}\right]
\end{aligned}
$$


where for computational convenience, we have rewritten $f(r)$ as $r g(r)$. Around $p_{2}$, with $z=u / v+1$, the $x_{2}$-direction turns out to be more convenient

$$
\begin{aligned}
x_{1}= & z, \quad x_{2}=-1+\omega+z-\frac{\epsilon r g(r)}{27(-1+\omega)}, \quad x_{3}=-1+\omega^{2}+z, \\
x_{4}= & a(-1+z), \quad x_{5}=c(-1+z)+b(-1+z)^{4} \\
& +\epsilon\left[\frac{-r}{3(b-a \psi) z}+\frac{7 r g(r)}{3(-1+\omega)(b-a \psi)}\right] .
\end{aligned}
$$

Finally, for $p_{3,4}$, we use $z=u / v-u_{*}$, where $u_{*}$ is one of the two roots of $u^{2}-u+1=0$, and we again go in the $x_{1}$-direction

$$
\begin{aligned}
x_{1}= & 1+u_{*}+z-\frac{\epsilon r g(r)}{27\left(1+u_{*}\right)}, \quad x_{2}=\omega+u_{*}+z, \quad x_{3}=\omega^{2}+u_{*}+z, \\
x_{4}= & a\left(u_{*}+z\right), \quad x_{5}=c\left(u_{*}+z\right)+b\left(u_{*}+z\right)^{4} \\
& +\epsilon\left[\frac{-r}{3(b-a \psi) z}+\frac{7 r g(r)}{3(b-a \psi)\left(1+u_{*}\right)}\right] .
\end{aligned}
$$

By construction, the restriction of $W$ to each of the tubes takes the form

$$
\left.W\right|_{T_{\epsilon}(C)} \sim \epsilon\left(r+r g(r)+\mathcal{O}\left(z^{2}\right)\right)+\mathcal{O}\left(\epsilon^{2}\right)
$$

thereby exhibiting the order of pole of each of the terms in (A.4). The convenience of choosing the plane in the $x_{5}$-direction becomes apparent when restricting the three-forms $\omega_{i}$ from (A.4). All terms in (A.3) involving $\omega_{5}$ vanish as a result of our choice. As for the non-vanishing three-forms, we have around $p_{1}$, for example,

$$
\begin{aligned}
& \omega_{2}=\frac{a+a \omega}{b-a \psi} z^{-1} \epsilon r^{2} g^{\prime}(r) d z d \epsilon d r, \\
& \omega_{3}=\frac{a \omega}{b-a \psi} z^{-1} \epsilon r^{2} g^{\prime}(r) d z d \epsilon d r \\
& \omega_{4}=\frac{-1-2 \omega}{b-a \psi} z^{-1} \epsilon r^{2} g^{\prime}(r) d z d \epsilon d r .
\end{aligned}
$$

The algebraic calculation of the residues is then accomplished by expanding (A.14) to the order in $\epsilon$ and $z$ dictated by the pole order of the three-form under consideration, picking out the term of degree 0 , and integrating over $r$. We do this around each of the four points (A.10), and sum up the results. 
For $f_{\text {exact }}$, we find, corresponding the 37 terms in (A.4)

$$
\begin{aligned}
& \text { (A.16) } \\
& 0+0+0+\frac{2\left(-31 a b \psi^{4}-62 a b \omega \psi^{4}+93 \psi^{5}+186 \omega \psi^{5}-5 a b \psi^{6}-10 a b \omega \psi^{6}+15 \psi^{7}+30 \omega \psi^{7}\right)}{3\left(-7+\psi^{2}\right)^{3}} \\
& +\frac{5\left(-7 a b \psi^{4}-14 a b \omega \psi^{4}+21 \psi^{5}+42 \omega \psi^{5}-2 a b \psi^{6}-4 a b \omega \psi^{6}+6 \psi^{7}+12 \omega \psi^{7}\right)}{3\left(-7+\psi^{2}\right)^{3}} \\
& -\frac{7\left(-a b \psi^{6}-2 a b \omega \psi^{6}+3 \psi^{7}+6 \omega \psi^{7}\right)}{\left(-7+\psi^{2}\right)^{3}}+0+0+0+0+0+0+0+0 \\
& +\frac{7\left(-a b \psi^{4}-2 a b \omega \psi^{4}+3 \psi^{5}+6 \omega \psi^{5}\right)}{3\left(-7+\psi^{2}\right)^{2}}+0+0+\cdots .
\end{aligned}
$$

For $f_{\text {direct }}$, we first have to calculate $n_{\psi}$ by differentiating the parameters entering the tube (which depend implicitly on $\psi$ ). We then contract $n_{\psi}$ with $\partial_{\psi}^{j} \tilde{\Omega}$ and feed the result into (A.7). In the case at hand, $f_{\text {exact }}$ turns out to vanish. Remembering some overall factors, and simplifying judiciously, the final result becomes precisely (2.29).

\section{References}

[1] J. Walcher, On the arithmetic of D-brane superpotentials. Lines and conics on the mirror quintic, Comm. Number Theory Phys. 6(2) (2012), 279-337, arXiv:1201.6427 [hep-th].

[2] G. Laporte and J. Walcher, Monodromy of an inhomogeneous PicardFuchs equation, SIGMA 8 (2012), 056, arXiv:1206.1787 [hep-th].

[3] D.R. Morrison, Picard-Fuchs equations and mirror maps for hypersurfaces, in Essays on Mirror Manifolds (S.-T. Yau, ed.), International Press, Hong Kong, 1992, (reprinted in: Mirror Symmetry I (S.-T. Yau, ed.), International Press, Cambridge, 1998, pp. 185-199), pp. 241-264, arXiv: hep-th/9111025.

[4] A. Klemm and S. Theisen, Considerations of one modulus Calabi-Yau compactifications: Picard-Fuchs equations, Kahler potentials and mirror maps, Nucl. Phys. B 389 (1993), 153, arXiv:hep-th/9205041.

[5] P. Candelas, X.C. De La Ossa, P.S. Green and L. Parkes, A pair of Calabi-Yau manifolds as an exactly soluble superconformal theory, Nucl. Phys. B 359 (1991), 21.

[6] D.R. Morrison, Mirror symmetry and rational curves on quintic threefolds: a guide for mathematicians, J. Amer. Math. Soc. 6 (1993), 223247, arXiV:alg-geom/9202004. 
[7] P. Deligne, Local behavior of Hodge structure at infinity, in 'Mirror symmetry', eds. B. Greene and S.T. Yau, AMS and International Press, AMS/IP Stud. Adv. Math., 1, 1997, 683

[8] C.F. Doran and J.W. Morgan, Mirror symmetry and integral variations of Hodge structure underlying one-parameter families of Calabi-Yau threefolds, in 'Mirror symmetry V', eds. Noriko Yui, Shing-Tung Yau, and James D. Lewis, AMS and International Press, AMS/IP Stud. Adv. Math., 38, 2006, 517-537.

[9] M. Green, P. Griffiths and M. Kerr, Neron models and boundary components for degenerations of Hodge structures of mirror quintic type, in 'Curves and abelian varieties', ed. V. Alexeev, Contemp. Math., 465, American Mathematical Society, 2007, 71-145, http: //www . math. wustl . edu/ matkerr/GGK. pdf.

[10] M. Kontsevich, L. Katzarkov and T. Pantev, Hodge theoretic aspects of mirror symmetry, in 'From Hodge theory to integrability and TQFT', Proc. Sympos. Pure Math., 78, American Mathematical Society, 2008, 87-174.

[11] M. Kontsevich, Gamma genus, talk at Arbeitstagung Bonn, May 2013.

[12] C.F. Doran and M. Kerr, Algebraic cycles and local quantum cohomology, arXiv:1307.5902 [math.AG].

[13] D.R. Morrison and J. Walcher, D-branes and normal functions, Adv. Theor. Math. Phys. 13 (2009), 553, arXiv:0709.4028 [hep-th].

[14] D. Krefl and J. Walcher, Real mirror symmetry for oneparameter hypersurfaces, J. High Energy Phys. 0809 (2008), 031, arXiv:0805.0792 [hep-th].

[15] J. Walcher, Calculations for mirror symmetry with D-branes, J. High Energy Phys. 0909 (2009), 129, arXiv:0904.4905 [hep-th].

[16] M. Green, P. Griffiths and M. Kerr, Neron models and limits of Abel-Jacobi mappings, Compos. Math. 146 (2010), 288-366, http: //www . math. wustl. edu/ matkerr/GGK1.pdf.

[17] A. Albano and S. Katz, Lines on the Fermat Quintic Threefold and the infinitesimal generalized Hodge conjecture, Trans. AMS, 324(1) (1991), 353; van Geemen's families of lines on special quintic threefolds, Manuscripta Math. 70(2) (1991), 183. 
[18] A. Mustaţă, Degree 1 curves in the Dwork pencil and the mirror family, Math. Ann. 355 (2013), 97-130, arXiv:math/0311252.

[19] A. Mustaţă, A note on higher degree curves in the Dwork pencil of quintics, unpublished and private communication; see also S. Xu, Degree 2 curves in the Dwork pencil,' PhD dissertation, Ohio State University, 2008.

[20] P. Candelas1, X. de la Ossa, B. van Geemen and D. van Straten, Lines on the Dwork pencil of quintic threefolds, Adv. Theor. Math. Phys. 16 (2012), 1779-1836, arXiv:1206.4961 [hep-th].

[21] A. Schwarz, V. Vologodsky and J. Walcher, preprint to appear.

Institute for TheOretical Physics

UNIVERSITEIT VAN AMSTERDAM

1090 GL AMSTERDAM

The Netherlands

E-mail address: R.A.Jefferson@uva.nl

Department of Physics and Department of Mathematics and Statistics MCGILL UNIVERSITY

Montreal, Quebec H3A 0B9

CANADA

E-mail address: johannes.walcher@mcgill.ca

ReCEIVED OCTOBer 22, 2013 